\title{
Modelling the Effect of Electrical Harness on Microvibration Response of Structures
}

\author{
Marcello Remedia, Guglielmo S. Aglietti \\ University of Surrey, Guildford, GU2 7XH, UK, m.remedia@surrey.ac.uk, Tel: +44(0)1483686029 \\ Guy Richardson \\ Surrey Satellite Technology Limited, Guildford, GU2 7YE
}

\begin{abstract}
The term "microvibrations" generally refers to accelerations in the region of micro g, occurring over a wide frequency range, up to say $500-1000 \mathrm{~Hz}$. The main issues related to microvibrations are their control and minimisation, which requires their modelling and analysis. A particular challenge is posed in the mid-frequency range, where many of the micro-vibration sources on board a spacecraft tend to act. In this case, in addition to the typical issues related to predicting responses in the mid-frequency, the low amplitude of the inputs can produce further non-linear behaviour which can manifest as uncertainties. A typical example is the behaviour of cables secured onto panels; when very low forces are applied, the presence of harness can influence the characteristics of the panel in terms of stiffness and damping values. In these circumstances, the cables themselves couple with the panel, hence become paths for vibration transmission. The common practise is to model such cables as nonstructural mass, however, this article illustrates that this method does not yield accurate results. In order to demonstrate this, an experimental campaign was conducted investigating a honeycomb panel, which was tested bare and with different configurations of harness secured to it. The results of this experimental campaign showed significantly different behaviour of the structure depending on the amplitude of the loads and the frequency. In particular, it was found that the effects the addition of the cables had on the panel were different depending on the frequency range considered. Based on this observation, a general methodology to deal with the whole frequency range is presented here and the basis to extend it to the case of more complex structures is also proposed.
\end{abstract}

Keywords: Harness, Mid-Frequency, Microvibration, Modelling Methodology, Craig-Bampton Stochastic Method

\author{
List of Abbreviations \\ FEM = Finite Element Model \\ $\mathrm{BP}=$ Bare Panel \\ TK_loc1 $=$ Panel with Thick Cable mounted in location 1 \\ TN_loc1 $=$ Panel with Thin Cable mounted in location 1 \\ TN_loc2 $=$ Panel with Thin Cable mounted in location 2
}


TKN $=$ Panel with Thick Cable mounted in location 1 and Thin Cable in location 2

FMCS $=$ Full Monte Carlo Simulation

CBSM = Craig-Bampton Stochastic Method

$\mathrm{SQM}=$ Structural Qualification Model

\section{Introduction}

Modern satellite payloads, e.g., the new generations of optical instruments, require extreme platform stability; hence microvibrations on board spacecraft have become an issue of growing importance. The term "microvibrations" generally refers to accelerations that can reach values as low as micro g, occurring over a wide frequency range, up to say $500-1000 \mathrm{~Hz}$. Research on microvibration started with the most advanced and sophisticated spacecraft, like the Hubble Space Telescope [1,2] or the earth imaging satellite SPOT [3, 4]. Nowadays, it is also a factor in the low cost end of the market, with microsatellites like the SSTL-150 platforms [5] or Skybox [6] which have the objective to carry cameras with very high pointing accuracy, and therefore even small relative displacements of the mounting points of the instrument, of the order of micro-meters, become unacceptable (as they produce large oscillations of the instrument line of sight). The research has focused on many aspects related to microvibrations, starting with the sources [7,8], and throughout the years various methodologies have been proposed for their modelling and control [9-12], e.g., simulating the interface between sources and spacecraft structures and predicting payload pointing errors [13, 14]. Among the difficulties related to microvibrations, one of the main ones is their analysis, having to deal with extremely low amplitudes $(\mu \mathrm{g})$ and a bandwidth that goes up to several hundred $\mathrm{Hz}$.

Problems are also found in terms of modelling, due to non-linearity, and of testing, as the noise from a standard test facility may exceed micro-g levels. Most of the equipment on board a spacecraft doesn't affect the linearity of the response. This means that the transfer function doesn't change when the amplitude decreases. On the other hand, when it comes to hardware such as braided elements (e.g., harness/cables or thermal straps) an increase of stiffness and decrees of damping are observed. The issue here is that for sufficiently high loads and deformations (e.g., during launch) the strands move/slide with respect to each other as the forces are higher than the friction forces between strands, so that the assembly has relatively low stiffness and high damping (the latter produced by the energy dissipated via the friction). Therefore, when high loads are applied, the contribution produced by stiffness of these elements is neglected and their mass contribution to the response is often represented in the Finite Element Model (FEM) simply as non-structural mass and higher damping; however when the loads are microvibrations this type of modelling is not accurate enough as the braided elements connected to the structure tend to behave like a monolithic element, hence producing a higher stiffness and lower damping. This article investigates the effect of breaded elements (i.e., multiple conductors' cables) on the structural response, and in particular non-linearity observed with respect to variations in the input loads. 
Many publications can be found on the influence of cables in other applications [15], e.g., bridges [16, 17], but only in the last few years, due to more challenging response prediction accuracy requirements, interest has been given to the modelling of cables in the context of spacecraft structure applications $[5,18,19]$. In particular, Goodding, Babuska et al. developed a method to study the behaviour of space structures supporting cables where the finite element model of the structure is updated with the addition of a model of the cable [20, 21]. The model updates required to represent the cable are calculated from a combination of: direct measurements, dynamic tests, static tension tests and wire handbook data (all this data is then fed into different tests to retrieve modulus of elasticity, shear modulus and damping) [22]; the cable attachments are also modelled. This work provided a good estimation of the effect the harness has on spacecraft structures and also gives excellent results for spacecraft panels with cables [22], however extending it to whole spacecraft structures would require a substantial modelling effort which is difficult to justify in the context of a commercial spacecraft project development. In addition, even when these relatively complicated modelling techniques were applied, it would still be necessary to extend them to reproduce the nonlinearities observed in the microvibration response and which are the studied in this article.

Consequently, the issue of extending the implementation of the effect cables have on more complex structures, without significantly increasing the modelling and computation effort is still open. This article proposes a methodology to deal with microvibrations acting on a simple structure which includes electrical cables and that can be easily extended to more complex structures.

\section{Methodology}

The development of this work followed a semi-empirical approach.

Firstly a test campaign was carried out to produce quantifiable evidence of the effect that braided elements (i.e., cables) have on the structures onto which they are fastened.

The test campaign considered a honeycomb panel, as typical spacecraft structural element, with different cables fixed in different locations. Various combinations were considered and the test item was subjected to inputs at various levels and in various locations.

The test results were then analysed methodically to identify specific trends, and a mathematical modelling technique able to envelop the effects of the presence of the cable (mass and stiffness), according to the frequency and magnitude of the inputs, was developed.

Finally the technique was extended to the case of a much more complex structural assembly.

\section{Experimental Campaign}

The equipment used for the test campaign were: an aluminium honeycomb panel of size $105 \times 71 \times 20 \mathrm{~mm}$ (cell size of $4.8 \mathrm{~mm}$, cell wall of 0.038 and core density of $0.07 \mathrm{~g} / \mathrm{cm}^{3}$ ); and two different braided cables, both $120 \mathrm{~mm}$ 
long: one with bundle diameter of $8 \mathrm{~mm}$ and one with bundle diameter of $12 \mathrm{~mm}$ (Figure 1). The braided cables could be situated in 2 different locations as represented in Figure 2: location 1 is the lower one built with 15 attachment points and location 2 is the upper one built with 9 attachment points. The five different configurations listed below were tested:

- $\quad$ Bare Panel (BP) [Figure 3]

- Panel with Thick Cable mounted in location 1 (TK_loc1)

- Panel with Thin Cable mounted in location 1 (TN_loc1)

- Panel with Thin Cable mounted in location 2 (TN_loc2)

- Panel with Thick Cable mounted in location 1 and Thin Cable in location 2 (TKN) [Figure 2]

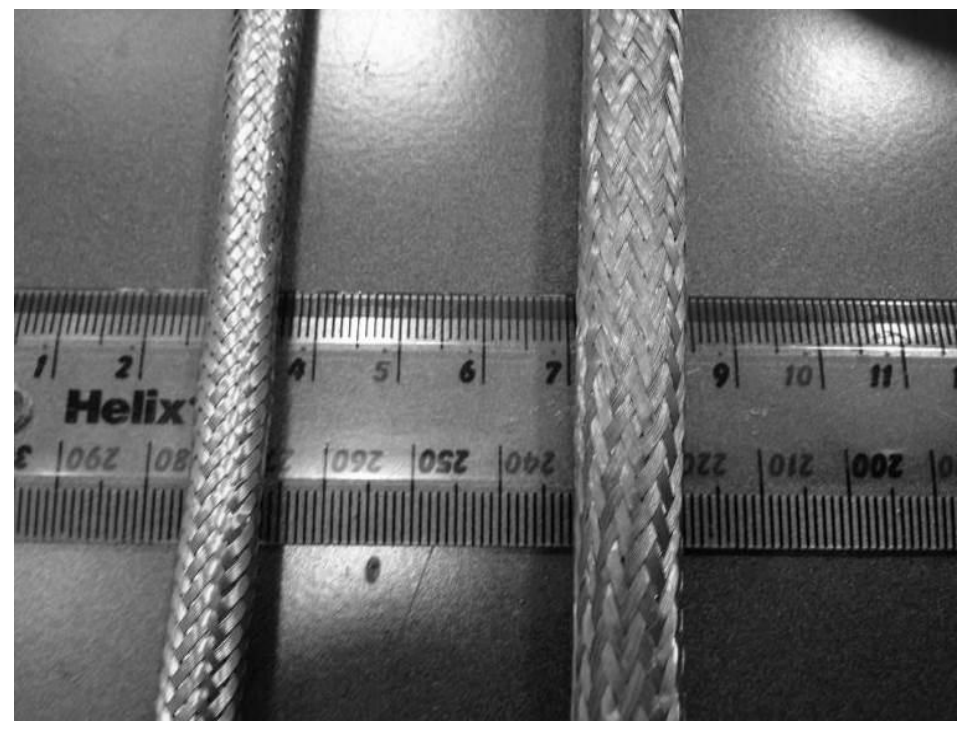

Figure 1: Cables used for the test campaign

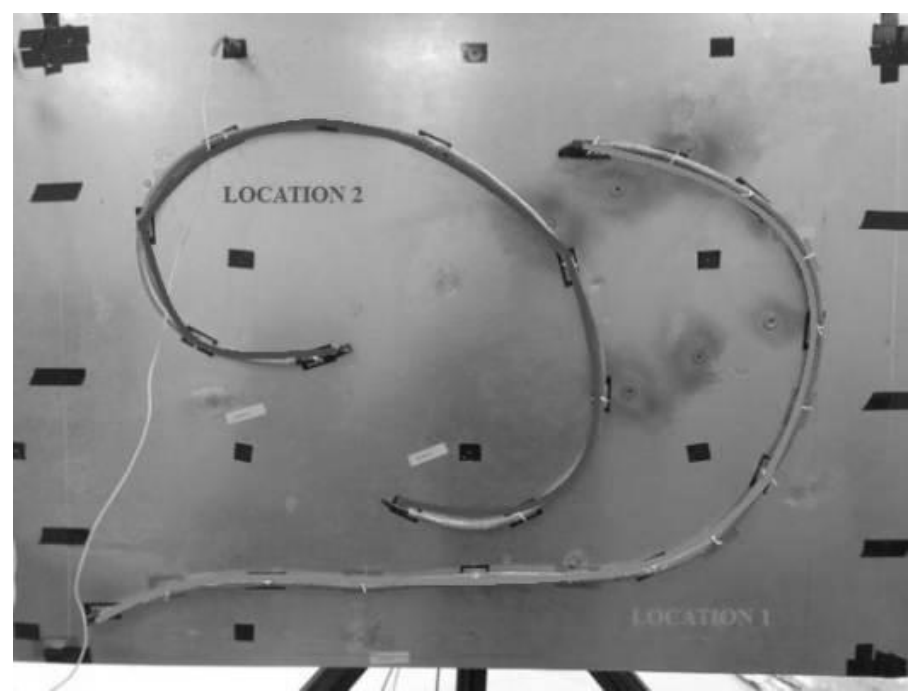

Figure 2: Locations where the harness was attached to the panel 
In all the configurations, a mini-shaker LDS V101 - max force $3 \mathrm{~N}$, was used to produce a force in a specific location and accelerometers with sensitivity of $1000 \mathrm{mV} / \mathrm{g}$ were used to acquire the response in 20 different locations. A controller software was used to create a sweep sine between $20 \mathrm{~Hz}$ and $1000 \mathrm{~Hz}$ to be used as input to the mini-shaker and 3 different levels of force were considered: $0.5 \mathrm{~N}, 0.05 \mathrm{~N}$ and $0.005 \mathrm{~N}$.

Having to deal with very low input forces, all the tests were performed in a very quiet environment in order to avoid any effect of the surroundings on the testing.

The honeycomb panel was suspended from elastic cords to reproduce free-free conditions, as it is shown in Figure 3. It can be observed that the panel has a 4 x 5 grid of pieces of Kapton tape attached to it: they identify the locations where the accelerometers were placed to acquire the responses. The 15 clips forming a curved shape were used to fasten the thick cable to the panel for location 1.

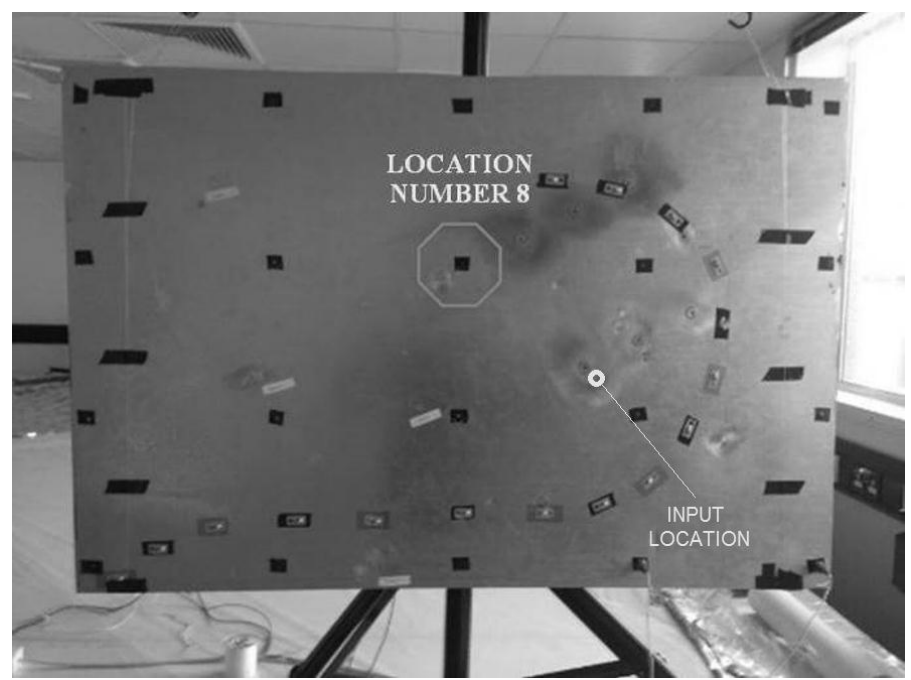

Figure 3: Honeycomb Panel suspended by elastic cords in order to reproduce free-free conditions

The mini-shaker was attached to the other side of the panel and it was driven by an amplifier connected to vibration controller software. The mini-shaker used is shown in Figure 4 and one of the accelerometers used is depicted in Figure 5.

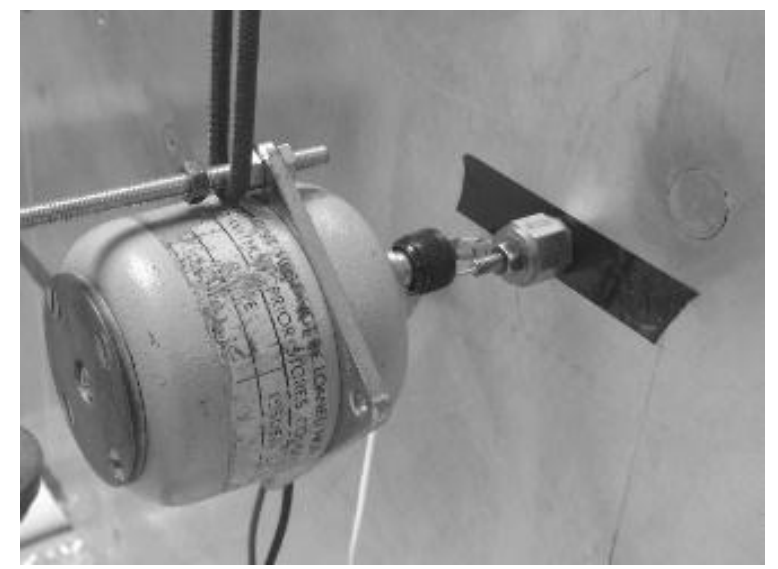

Figure 4: Mini-shaker used to reproduce forces in a specific location of the panel 
Figure 4 also shows the force transducer (with a measurement range of $10 \mathrm{lb}$ and sensitivity of $500 \mathrm{mV} / \mathrm{lb}$ ) that was used with the mini-shaker, connected with a "stinger" (which has high longitudinal stiffness and negligible lateral and bending stiffness so that the shaker imparts a point force). The transducer also worked as feedback control signal for the testing: the signal registered by the force transducer was sent back to the controller, which adjusted the amplitude of the input produced in order to maintain it constant.

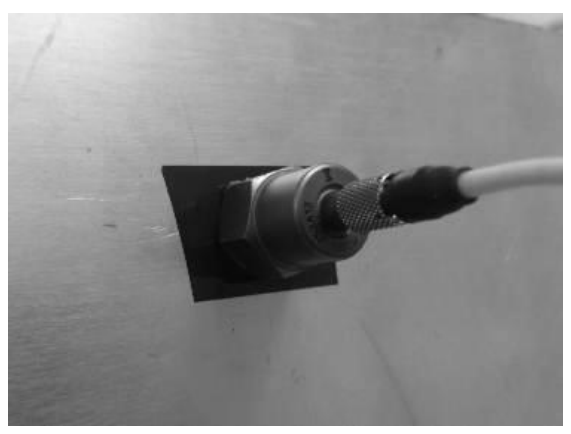

Figure 5: Accelerometer type used during the experimental campaign

Firstly, an acquisition of the noise was performed before every test in order to confirm that the level of magnitude was at least 2 - 3 orders of magnitude lower than the signals acquired. This was done in terms of Power Spectral Densities (PSDs): a PSD of the noise acquisition (a Hamming window was used with a 50\% overlap and a sampling rate of 2500 samples/sec) was compared to the PSD of the signal produced by the mini-shaker. Once the noise was confirmed not to be an issue, all the acquisitions were measured as frequency response functions. The first tests were performed on the BP: the acquisition was made with the same software controlling the input.

In a second phase, the thick cable was attached to the panel, using the 15 locations shown in Figure 3, as shown in Figure 6.
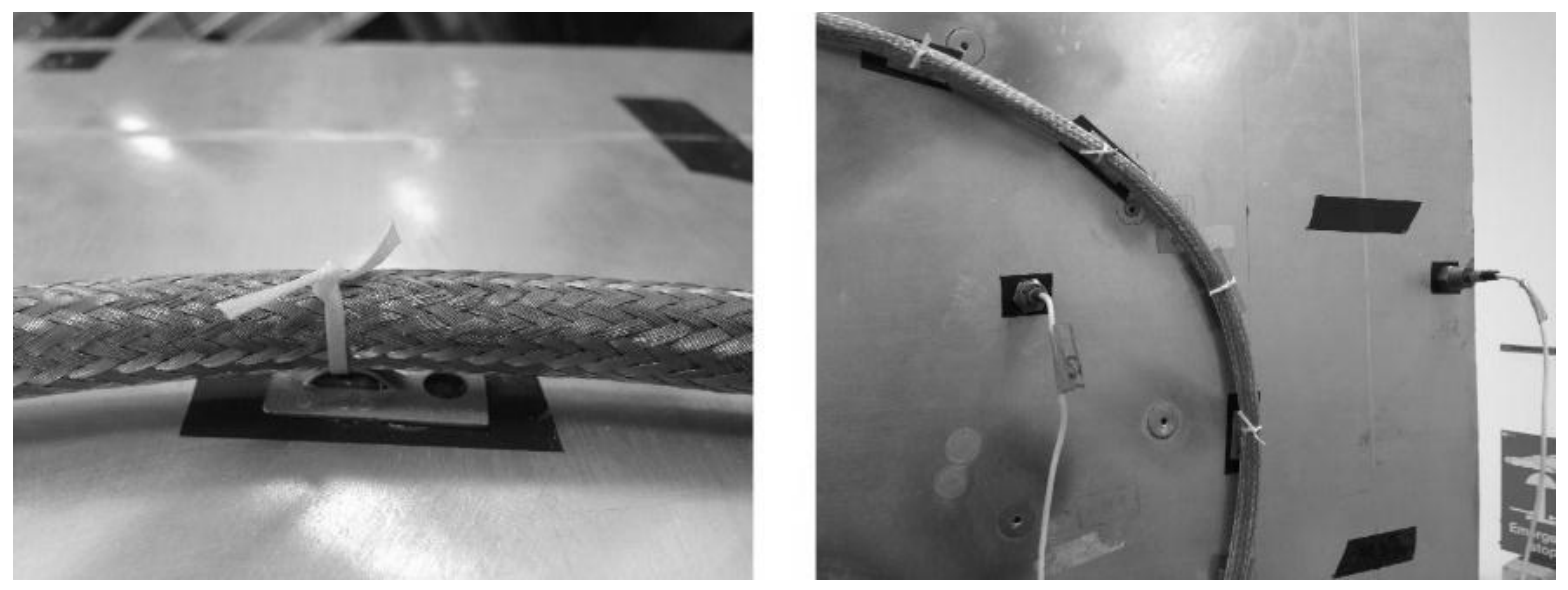

Figure 6: Details of the thick cable attached to the panel

The same test campaign adopted for the BP was repeated for the TK_loc1 configuration. The same procedure was then also applied for the other three configurations (TN_loc1, TN_loc2 and TKN). In the next section the results of the experimental campaign here described will be shown and discussed. 


\section{Experimental Results}

The results of the experimental campaign will be here shown using different comparisons in order to describe different aspects important for the analysis approach to be taken and developed in Section V. In particular, three features will be considered:

- the variation of the input level;

- the variation of the cable thickness;

- The effect of the location where the cables are attached to the panel.

In order to maintain a coherent development throughout the article, the outputs from location number 8 were selected as example results for display here, as they are representative of the trends seen for all output locations (Figure 3). The description of the experimental and computational campaign is developed referring to specific frequency ranges: in this specific case with low-frequency range we refer to 0-300 Hz, mid-frequency $300-600 \mathrm{~Hz}$ and high-frequency 600-1000 Hz; but more in general the low-frequency range includes the first few resonances of the structure considered, the high-frequency range starts when a consistent modal overlap can be observed and the mid-frequency range everything in the middle.

The first feature to be assessed is the change in response resulting from the variation of the input level. In order to investigate this, three different configurations were chosen: BP, TK_loc1 and TKN. As expected, in Figure 7(a) (BP configuration) the transfer functions for all the inputs don't significantly change, showing linearity, which is characteristic when low inputs are applied to structures which don't include braided elements. On the other hand, when the cable is attached to the panel, lowering the loads produces a significant difference (see Figure 7(b)): in the mid-frequency range the response changes and the second resonant frequency looks particularly damped when a higher force $(0.5 \mathrm{~N})$ is applied; the same effect doesn't happen for the following peaks (the same behaviour is also observed in all the other outputs, but it is here omitted). The same trend is even more noticeable with the TKN configuration (see Figure 7(c)).

The second feature to be analysed is the change in response incurred by the variation of the cable thickness. In order to assess this, the BP, TK_loc1 and TN_loc1 configurations were compared for the different input levels. Looking at the low-frequency range of the graph depicted in Figure 8(a), the effect of the damping is significantly stronger than in the other cases (Figure 8(b) and Figure 8(c), see Table 1): for high loads the cable strands create friction and therefore high damping; at lower loads cables behave like a monolithic structure therefore giving more stiffness and less damping. The context of this paper refers to a microvibration environment and therefore the focus will be on the lower loads. The first three peaks, which is what is considered low-frequency in this article, look consistent: a lower frequency given by an increase of the mass (i.e., the mass of the cable), but damped compared to when the panel was tested without any piece of harness attached to it. When the frequency increases beyond the first few resonances, the response looks completely different and it is difficult to observe a pattern that can be attributed to the presence of the cable. Finally, at high frequencies the results obtained in the two cases tend to be very similar, which allows using the same method used for the modelling of the structure without harness attached to it without further modifications. 


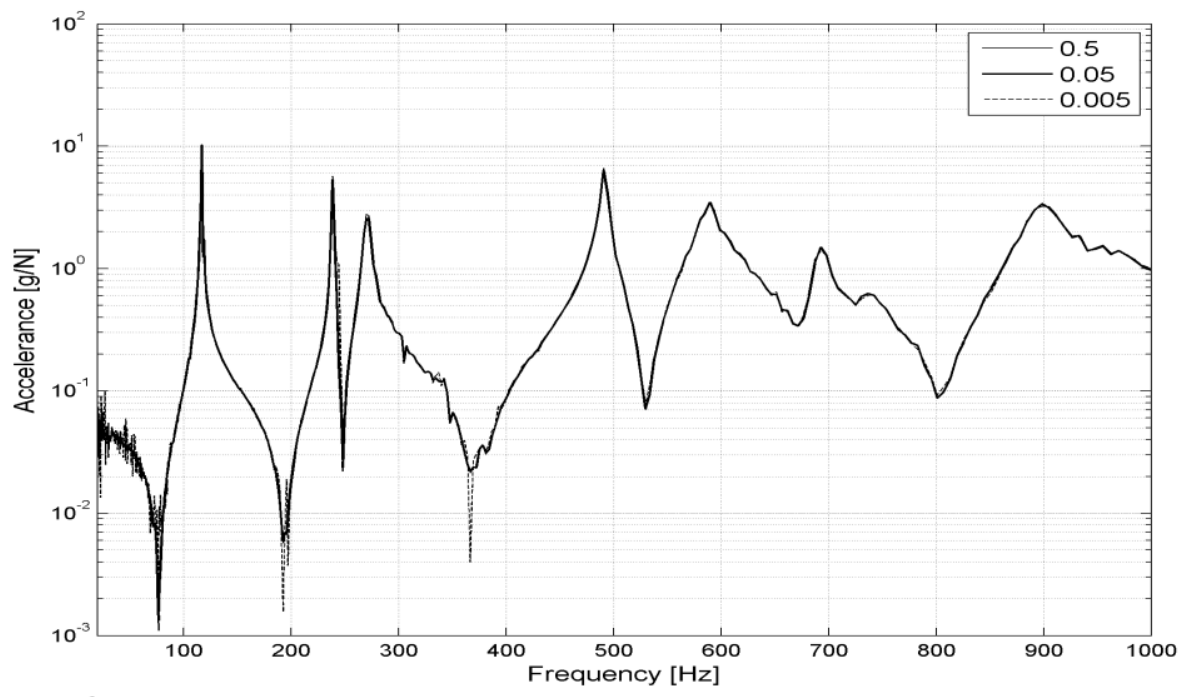

(a)

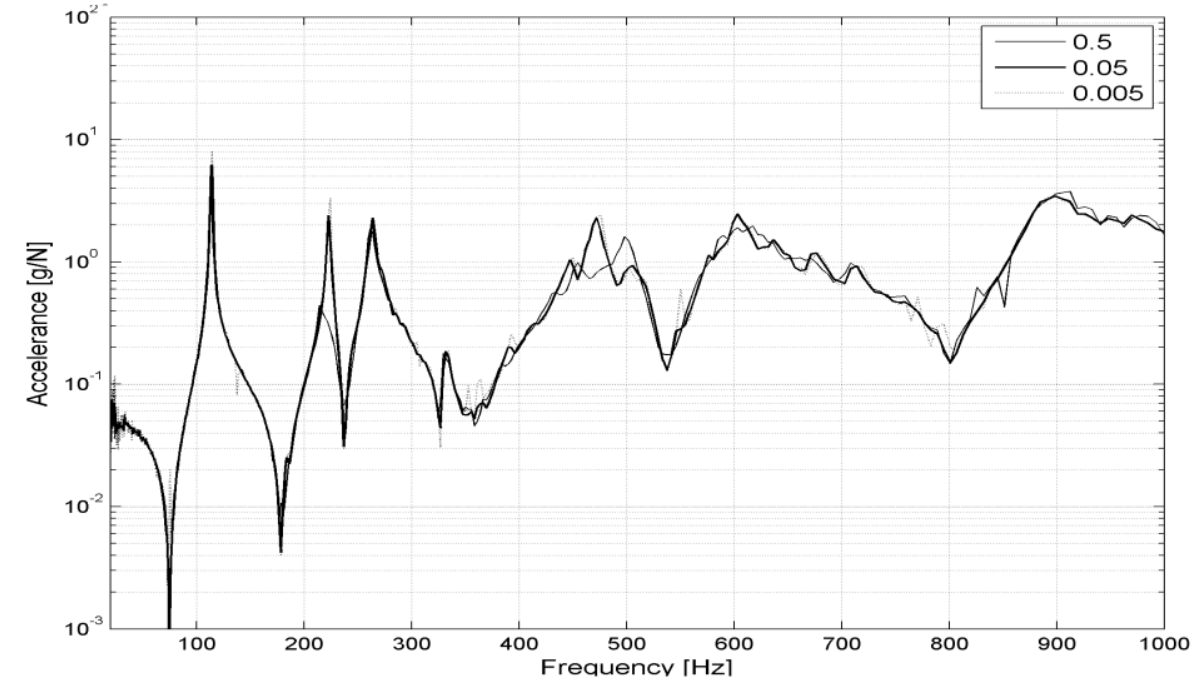

(b)

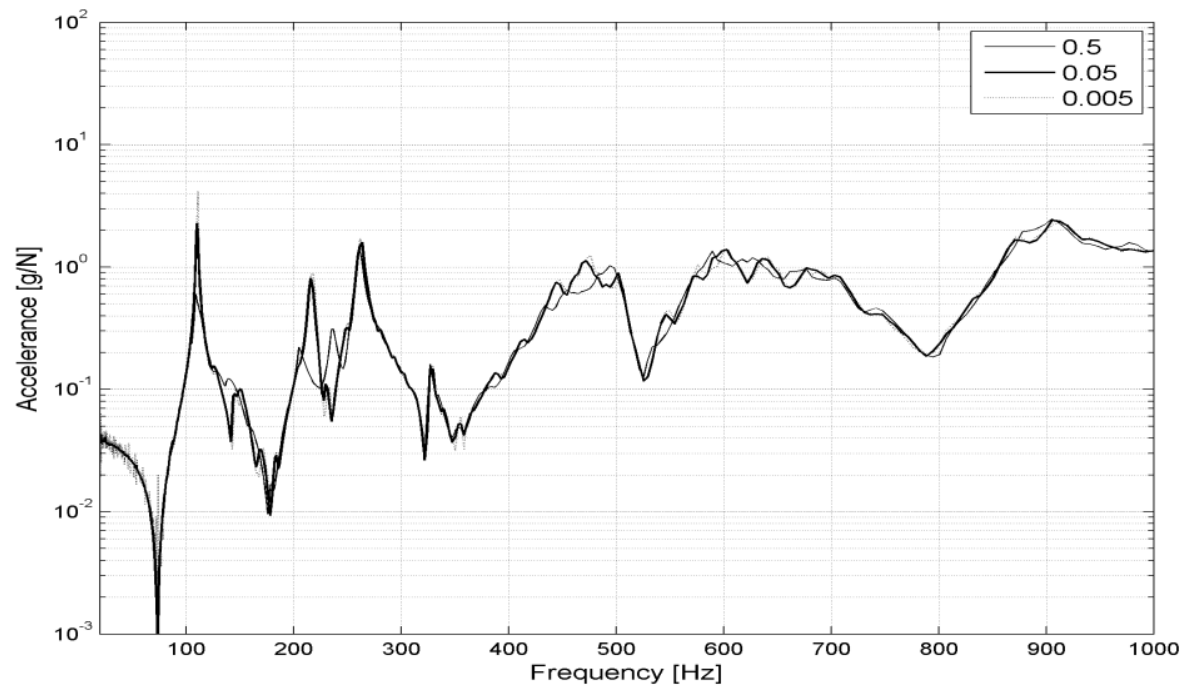

(c)

Figure 7: Comparison amplitudes transfer functions of the different inputs applied for (a) BP, (b) TK_loc1 and (c) TKN configurations. 


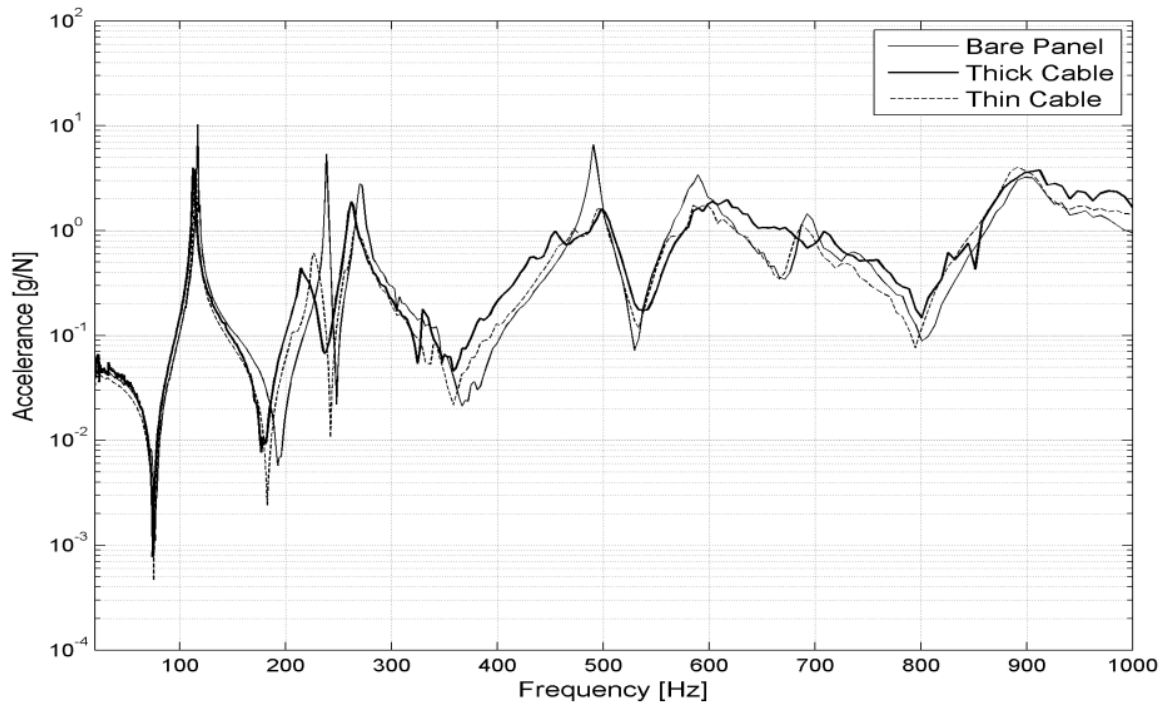

(a)

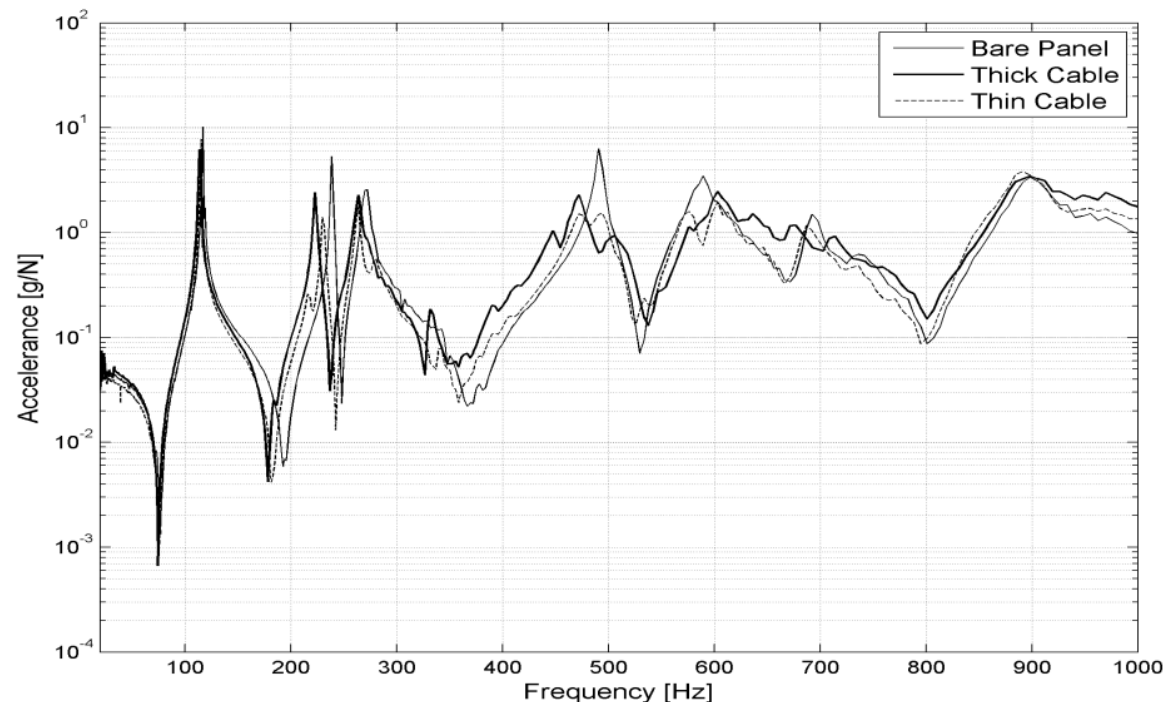

(b)

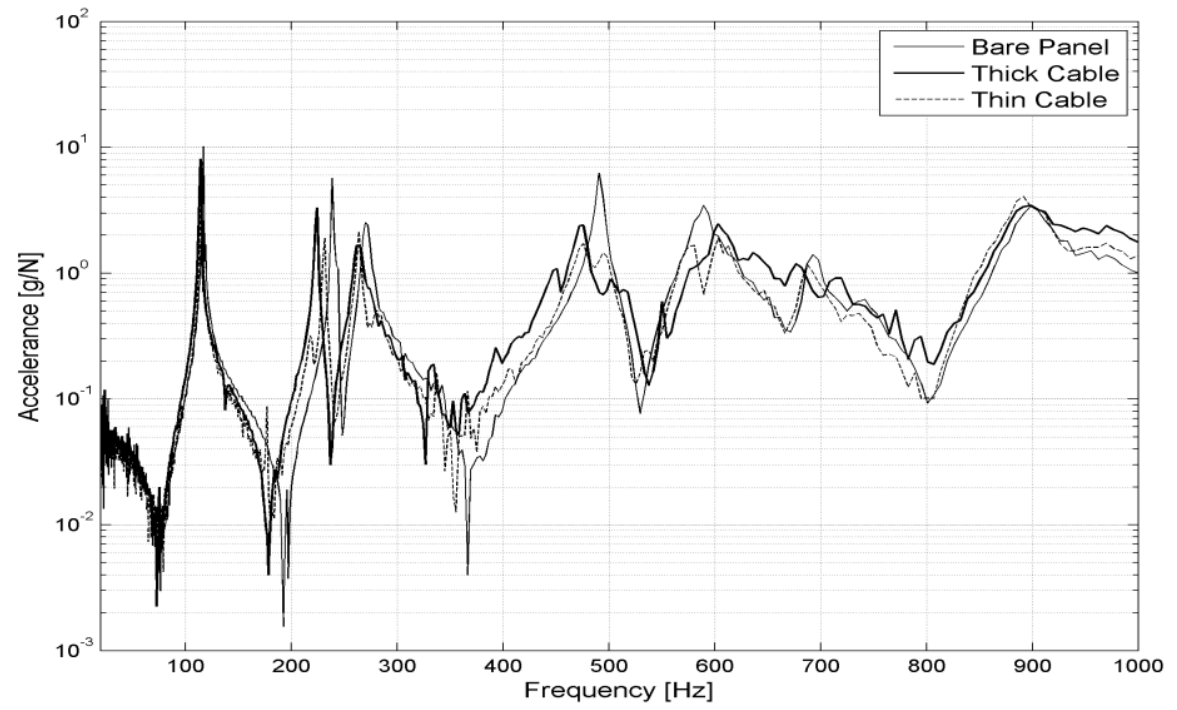

(c)

Figure 8: Comparison transfer functions with input (a) $0.5 \mathrm{~N}$, (b) $0.05 \mathrm{~N}$ and (c) $0.005 \mathrm{~N}$ for BP, TK_loc1 and TN_loc1 configurations 


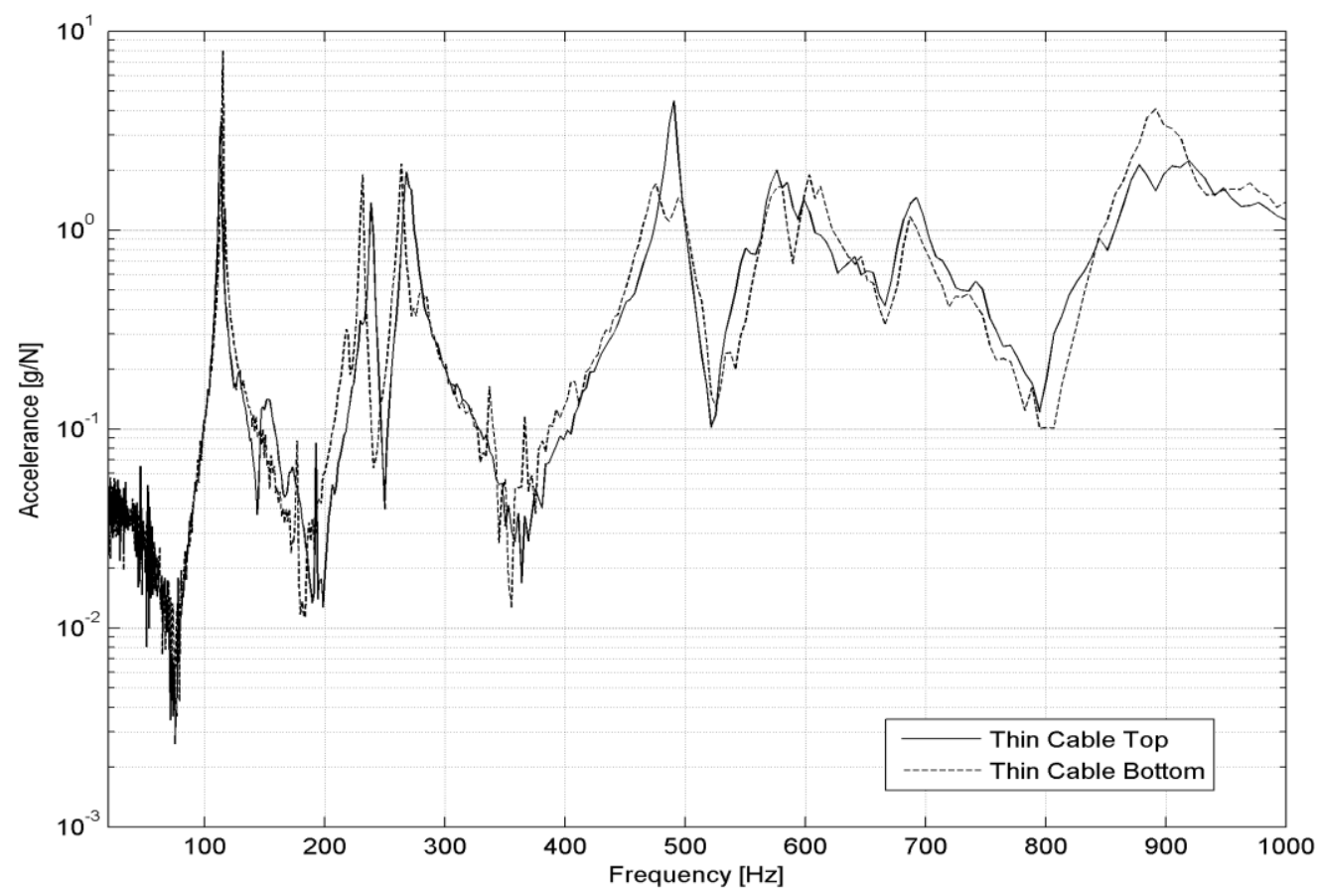

Figure 9: Comparison transfer functions with input $0.005 \mathrm{~N}$ for $\mathrm{TN} \_l o c 1$ and $\mathrm{TN} \_l o c 2$ configurations

Table 1: Damping values for the peak at $\sim 230 \mathrm{~Hz}$ calculated with the half-power method

\begin{tabular}{cccc}
\hline & $\mathbf{0 . 5} \mathbf{~ N}$ & $\mathbf{0 . 0 5} \mathbf{~ N}$ & $\mathbf{0 . 0 0 5} \mathbf{~}$ \\
\hline Bare Panel & $0.31 \%$ & $0.31 \%$ & $0.31 \%$ \\
Thin Table (TN_loc1) & $1.5 \%$ & $0.47 \%$ & $0.53 \%$ \\
Thick Cable (TK_loc1) & $2.35 \%$ & $0.67 \%$ & $0.43 \%$ \\
\hline
\end{tabular}

Finally, the third feature to be analysed is the change in response which results from moving the location of the cable on the panel. Figure 9 shows the comparison between the outputs measured when the thinner cable was positioned in the two different locations of the panel (Figure 2). The graph shows a shift of the peaks slightly to the lower frequencies; the mass has not changed, but the location has, and the points where the cable is connected affect the coupled dynamics between the cable itself and the panel. Therefore, this represents further proof of the fact that modelling the harness with non-structural mass would not be accurate enough.

In conclusion, for low magnitude input loads the problem can be divided into three different stages:

- Low-frequency range: the presence of the cable produces an increase of the stiffness, which significantly counterbalances the decrease of the frequency resonances caused by the addition of mass; (overall the effect of the added mass is higher than that of the added stiffness, and the resonance frequencies of the panel with the cable(s) are lower than those of the bare panel) furthermore, higher damping when compared to the structure without cable is observed;

- Mid-frequency range: quite different behaviour, but in general more damped;

- High-frequency range: the presence of the cable does not significantly change the response (Figure 8).

These three stages are now the basis for the methodology that will be developed in the next section. 


\section{Computational Approach}

Following the trends shown during the experiments the analytical approach can be divided in three parts; for the low, medium and high frequency ranges. For the high frequency range, the predictions are always very similar regardless of the presence of cables (i.e., the cable does not significantly change the response). Therefore, no further analyses need to be implemented and the same method used for the bare structure (e.g., Statistical Energy Analysis) can be utilised also for the structure with cables. This is in line with the rational that modelling harness with NonStructural Mass would lead to erroneous predictions as at high frequencies we are above the cable fundamental modal frequencies and therefore their contribution to the response predictions can be neglected, hence the same behaviour of the panel with and without the piece of harness attached to it. Therefore, the focus here will be on the low- and the mid-frequency ranges. In the first case, a significant change will be addressed; in the latter case a Full Monte Carlo Simulation (FMCS) with a particular set for the damping seems to be the best choice and a different implementation will be described for the case of having to deal with more complex structures. The experimental test campaign also showed differences concerning the amplitude of the input transmitted: Table 2 recaps the influence harness has on the structure it is attached to for all the different cases.

Table 2: Influence of the harness according to input level and frequency range

\section{Low-Frequency Mid-Frequency High-Frequency}

\begin{tabular}{|c|c|c|c|}
\hline Microvibrations & $\begin{array}{l}\text { Harness changes the } \\
\text { response as contribution } \\
\text { to changes in mass, } \\
\text { stiffness and damping }\end{array}$ & $\begin{array}{l}\text { Harness resonances couple } \\
\text { with the structure ones and } \\
\text { create a very different } \\
\text { response, higher damping }\end{array}$ & $\begin{array}{l}\text { Above harness' fundame- } \\
\text { ntal modal frequencies and } \\
\text { therefore no influence is } \\
\text { observed in the response }\end{array}$ \\
\hline or & $\begin{array}{l}\text { Very consistent damping } \\
\text { variations are generated } \\
\text { compared to } \\
\text { microvibration levels }\end{array}$ & $\begin{array}{c}\text { The effect created for } \\
\text { microvibration level is less } \\
\text { accentuated, higher } \\
\text { damping }\end{array}$ & $\begin{array}{l}\text { Above harness' fundame- } \\
\text { ntal modal frequencies and } \\
\text { therefore no influence is } \\
\text { observed in the response }\end{array}$ \\
\hline
\end{tabular}

\section{A. Finite Element Model}

Firstly, a Nastran FEM of the Bare Panel was built. Plane strain quadrilateral elements (CQUAD) were used to build the panel with PSHELL property to describe the honeycomb panel characteristics. MAT1 and MAT8 are the cards that were used as inputs to the PSHELL property. The FEM of the panel, with the input force and output locations highlighted in red and yellow respectively, is depicted in Figure 10. 


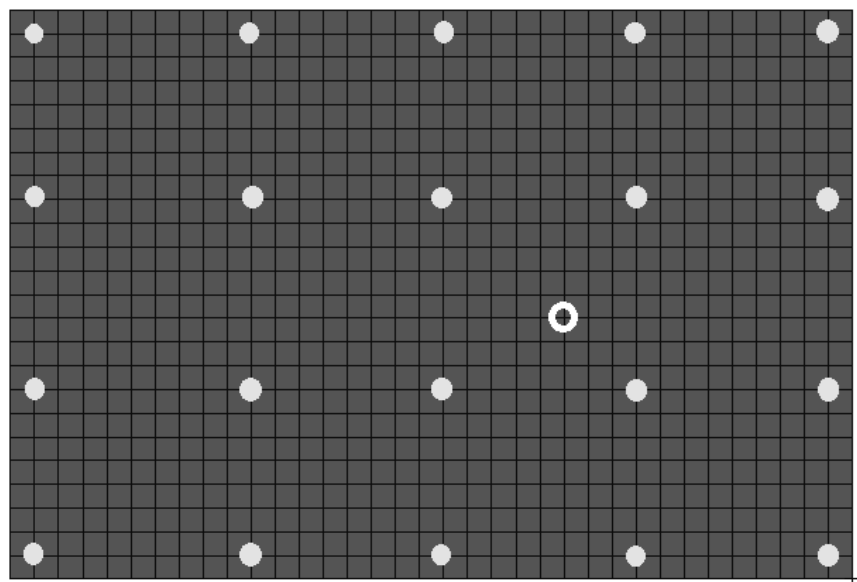

Figure 10: Finite Element Model of the Bare Panel with input and outputs highlighted in rings and filled circles, respectively

\section{COMPUTATIONAL MODE SHAPES}

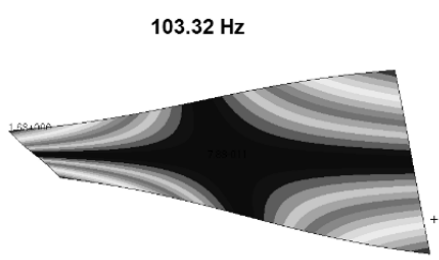

$267.8 \mathrm{~Hz}$

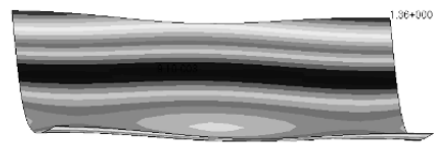

116.38 Hz

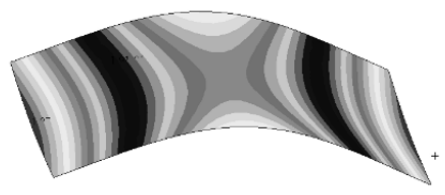

$297.08 \mathrm{~Hz}$

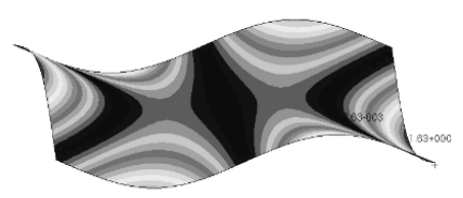

$237.27 \mathrm{~Hz}$

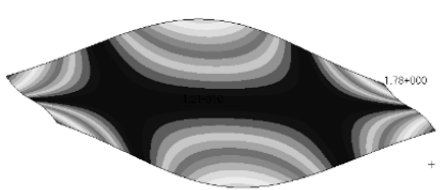

$348.36 \mathrm{~Hz}$

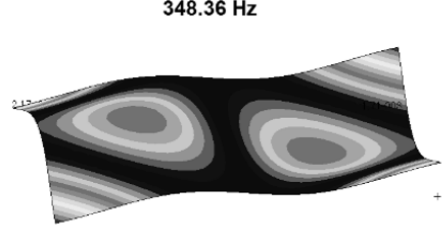

\section{EXPERIMENTAL MODE SHAPES}

$102.93 \mathrm{~Hz}$

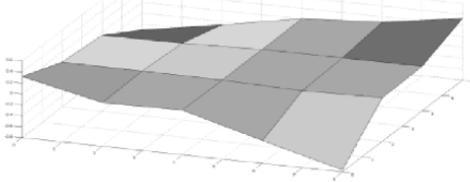

$267.99 \mathrm{~Hz}$

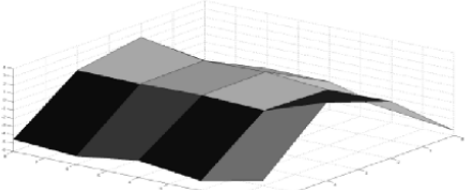

$116.34 \mathrm{~Hz}$

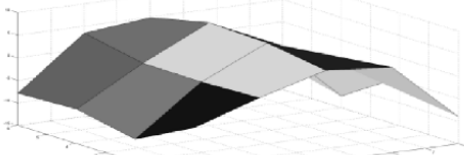

$300.61 \mathrm{~Hz}$

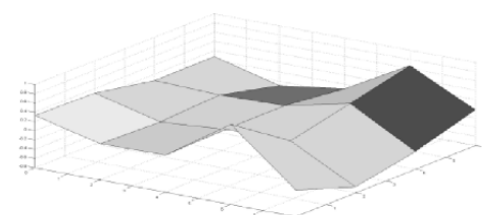

$237.10 \mathrm{~Hz}$

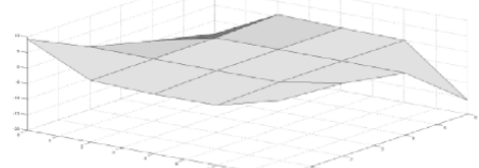

$353.03 \mathrm{~Hz}$

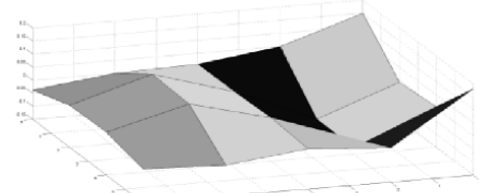

Figure 11: First 6 mode shapes of the Bare Panel: Computational (above) and Experimental (below) 
Figure 12 depicts a comparison of the transfer functions obtained from the test campaign and the one obtained using the FEM (shown in Figure 10). Figure 11 shows the comparison between the experimental and the computational mode shapes, a further confirmation of the validity of the FEM. Looking at Figure 11, the input lies very close to a nodal line when referring to the mode shape at $\sim 350 \mathrm{~Hz}$. That is the reason why there is a mismatch in Figure 12 between experimental result and prediction: in the experimental result the input was exactly located in the nodal line, whilst in the prediction it only was very close to it, and therefore a peak was produced.

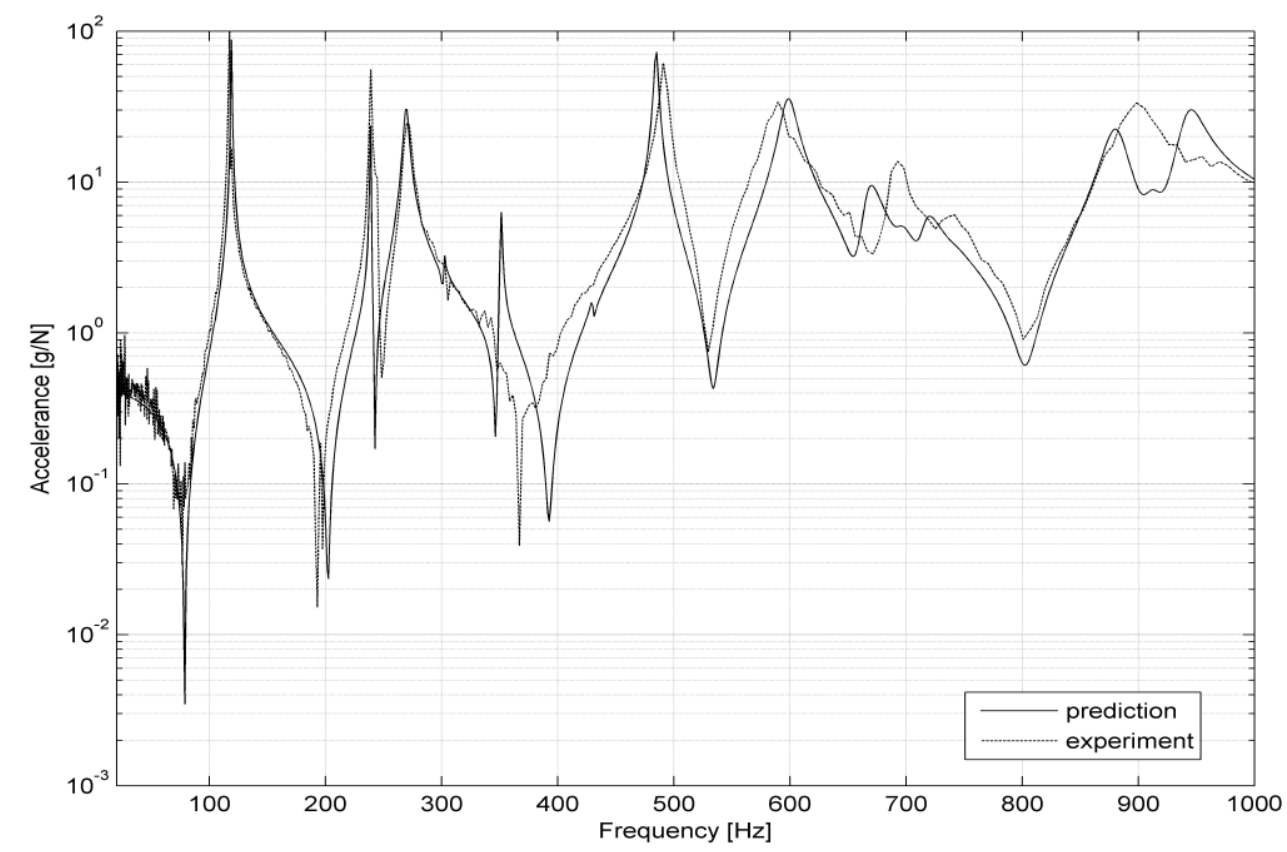

Figure 12: Comparison predictions and experimental results for bare panel

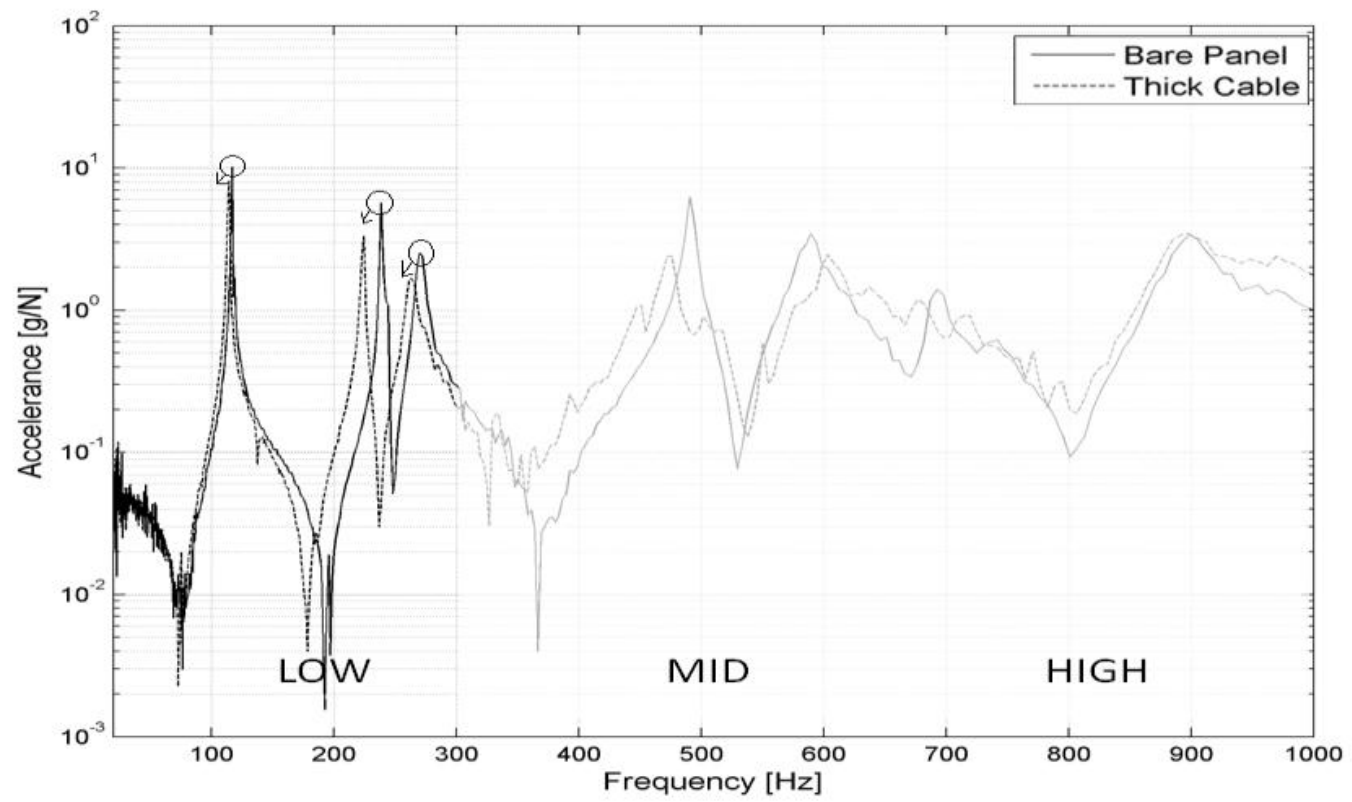

Figure 13: Comparison transfer functions with input $0.005 \mathrm{~N}$ for Bare Panel and Thick Cable configurations - focus in the low-frequency range 


\section{A. Low-Frequency Predictions}

The comparison between the BP and the TK_loc1 configurations is again shown in Figure 13, but this time focussing on the low-frequency range.

As can be seen from the graph in Figure 13, when the thick cable is present the first three peaks all tend to occur at a lower frequency and have blunter, less sharp peak than when the panel was bare. At first glance, it could be concluded that the damping increases with the frequency, whilst in order to decrease the frequency of the resonances, an addition of mass (as Non-Structural Mass) seems to be sufficient. In reality, it can be observed that the peaks are moving to lower frequencies differently and that suggests a change also in the stiffness.

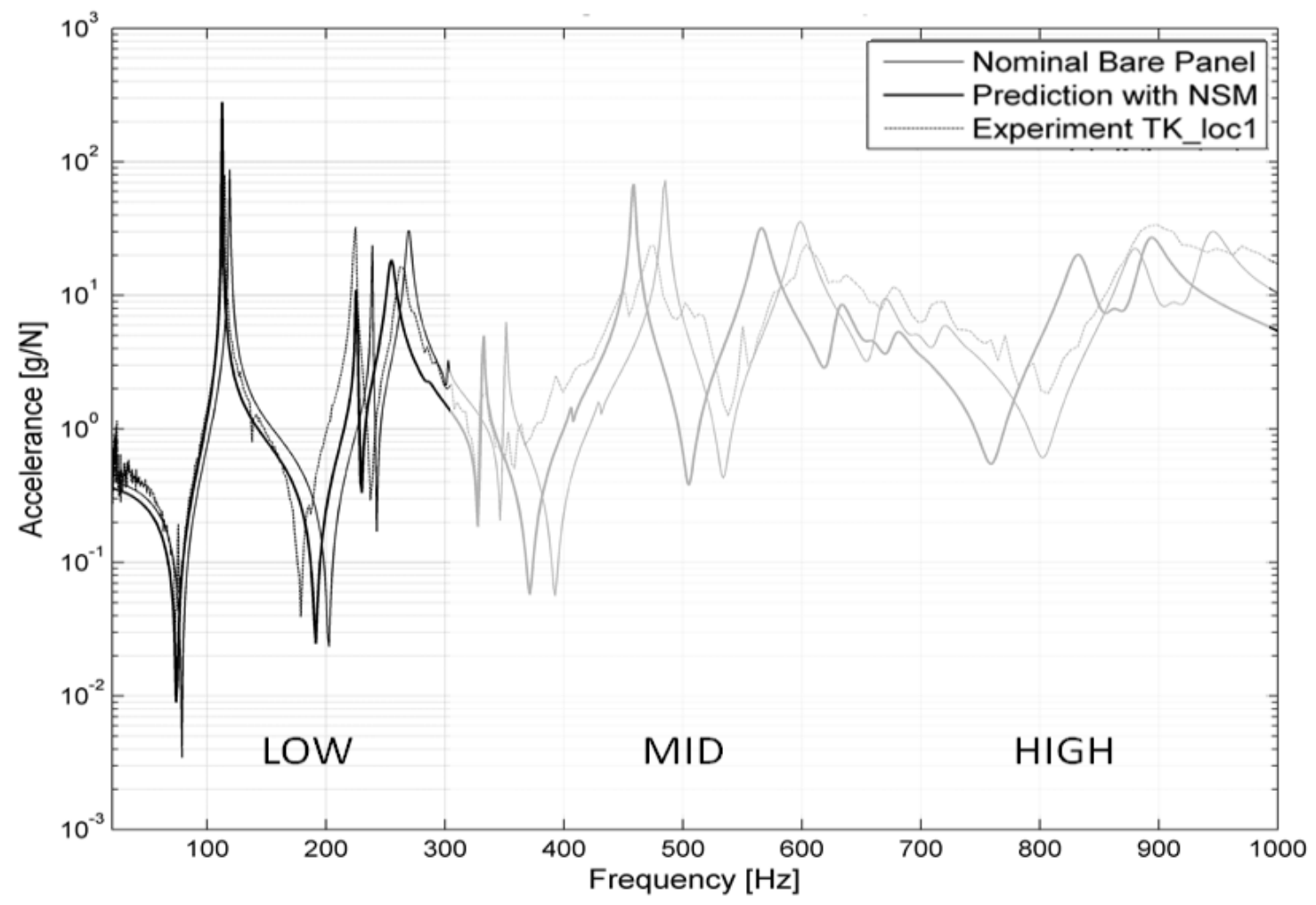

Figure 14: Prediction obtained adding Non-Structural Mass to represent the cable compared to the Bare Panel nominal prediction and the experimental result with the cable attached

Figure 14 shows the prediction that is obtained with only addition of Non-Structural Mass to represent the piece of harness (a mass equal to the weight of the harness was smeared onto the panel) and from the graph it is very clear that an addition of stiffness is also required to have the prediction peaks to match with the experimental results. This was also done (in particular with an increase of the stiffness of the main materials of the panel of $4.5 \%$ ) and it is shown in Figure 15. The increase of stiffness applied to this example is not to be taken as a guideline; this section only demonstrates that an increase of stiffness is needed: the magnitude of it is specific to each case.

From the graph in Figure 15 it can be observed that by adjusting the stiffness, the errors in the frequency of the first and third peaks decreased from $2.5 \%$ to values lower than $0.5 \%$. The second peak, on the other hand, is not 
affected by the change of stiffness in the cable location and already matched with the experimental results with the only addition of Non-Structural Mass: this is explained by the fact that the cable mostly lies on the nodal lines created by the mode of the panel at $237 \mathrm{~Hz}$ (see Figure 11) and therefore doesn't affect the original predictions obtained with the FEM of the BP.

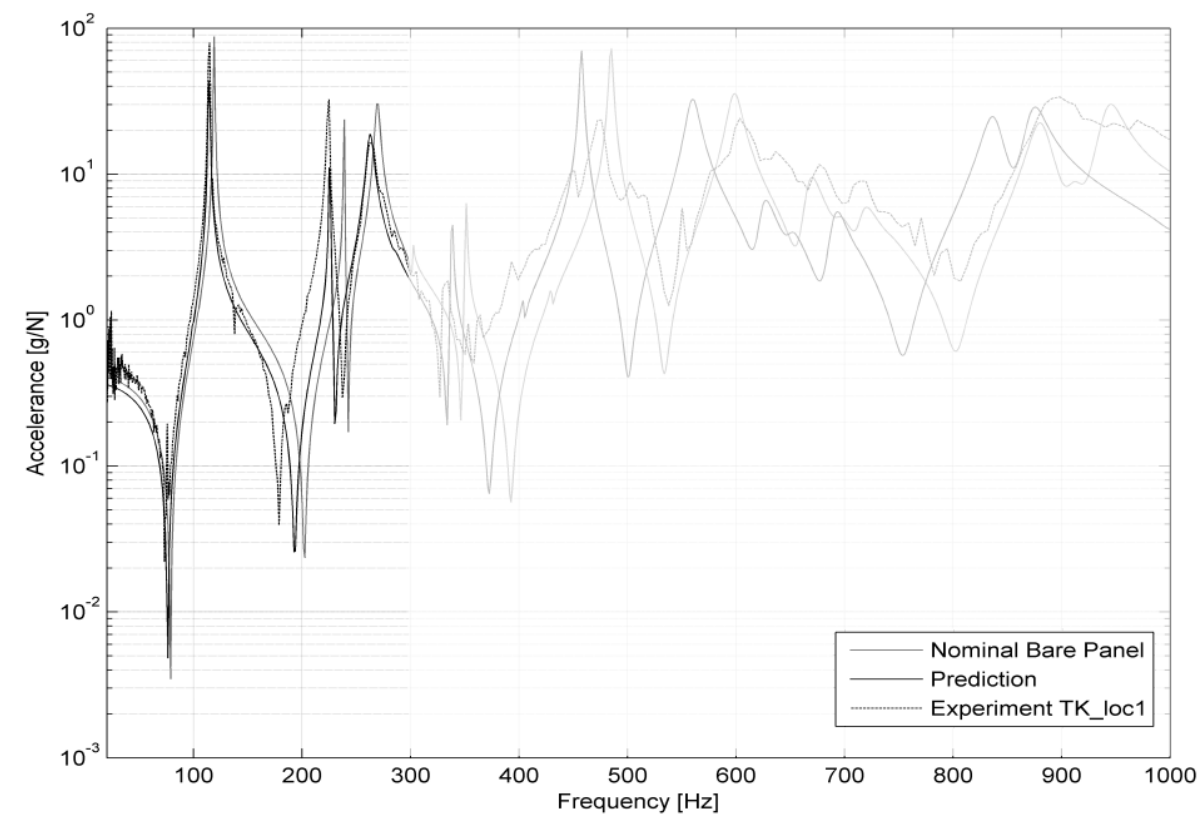

Figure 15: Prediction obtained adding Non-Structural Mass and stiffness to represent the cable compared to the BP nominal prediction and the experimental result with the cable attached

\section{B. Mid-Frequency Predictions}

The comparison between the BP and the TK_loc1 configurations is again shown in Figure 16, but this time focussing on the mid-frequency range.

In the mid-frequency range the situation is more complicated: the modes of the cables couple with the modes of the panel creating a consistent modal overlap which produces a response not as definite as it was in the lowfrequency range or with the $\mathrm{BP}$ configuration. Damping also plays an important role as the corresponding peaks of the BP configuration are now much less sharp. All this suggests a modelling technique that produces more significant changes to the predictions rather than only working on Non-Structural Mass and stiffness in the cable location. Here a FMCS was chosen to deal with the mid-range frequencies, from 300 to $600 \mathrm{~Hz}$ for this specific case. In the FMCS many samples of the stochastic system are generated using a random number generator and the final equilibrium is solved as many times, leading to a population of the response vector; based on this population, the response variability of the system is calculated using simple relationships of statistics. FMCS is a highly expensive approach from a computational point of view, which is still affordable for applications as simple as a single honeycomb panel, but less so for structures as complex as full spacecraft. In the next section a different approach will be therefore described, which merges the efficiency of the Craig-Bampton reduction technique with the simplicity and reliability of FMCS. 


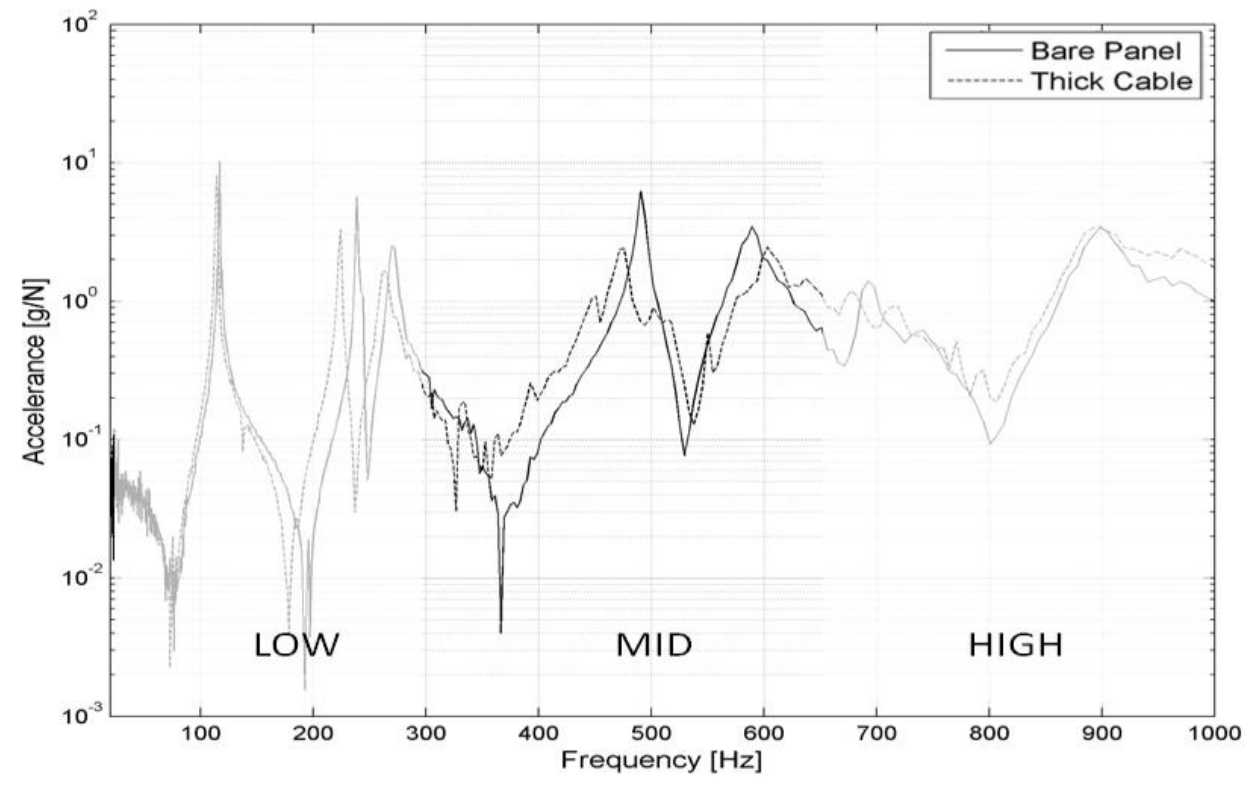

Figure 16: Comparison transfer functions with input $0.005 \mathrm{~N}$ for $\mathrm{BP}$ and $\mathrm{TK}$ _loc1 configurations - focus in the mid-frequency range

Starting from the model used to correct the low-frequency range, a Gaussian randomisation (with a $\sigma$ of $5 \%$ ) of the stiffness and mass properties of the elements of the panel was applied. One hundred different models were built, run and statistically manipulated (average summed to the standard deviation twice to obtain a $95 \%$ confidence curve) to obtain the final response. A new damping card was also produced with a significant increase of the values used so far. The results of this FMCS are shown in Figure 17.

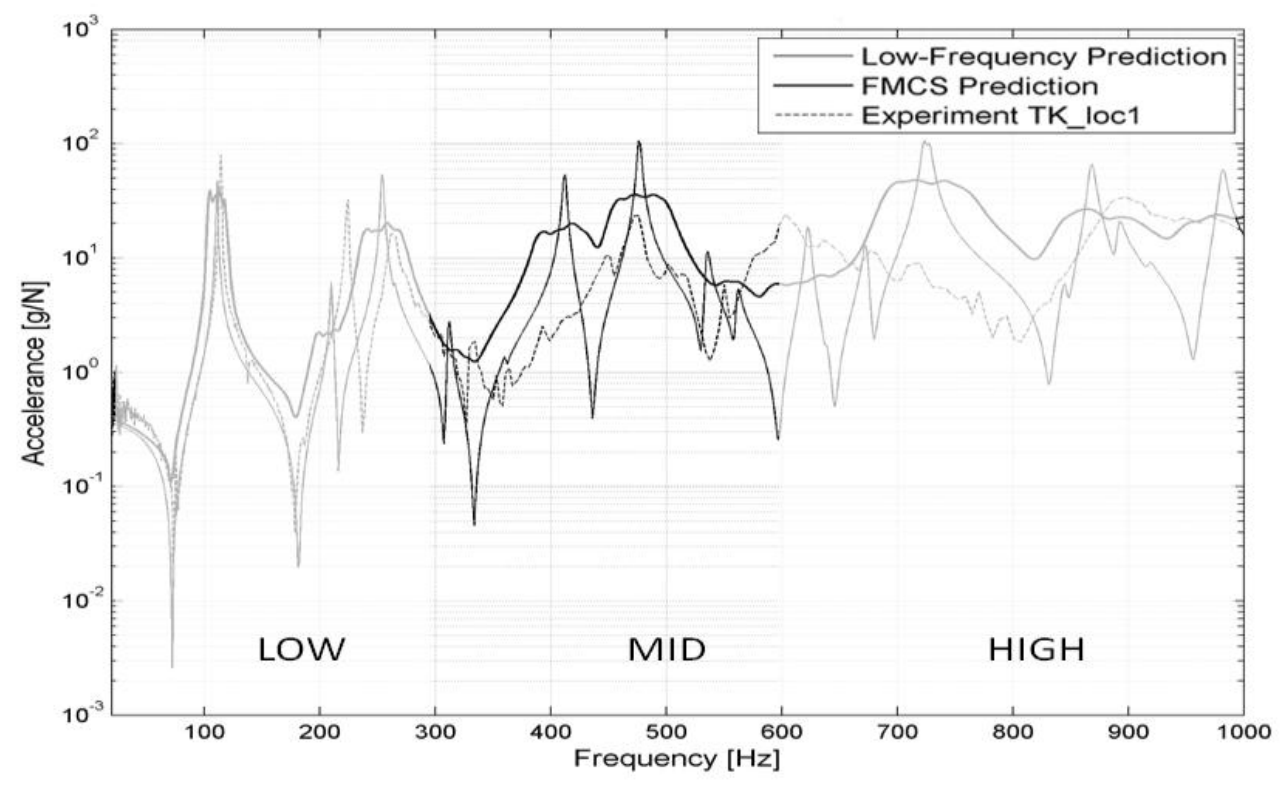

Figure 17: Prediction obtained through a FMCS compared to the low frequency TK_loc1 prediction and the experimental result with the cable attached 
The FMCS prediction shown in Figure 17 represents the upper limit of a confidence envelope: it is supposed to contain the worst case scenario produced by the modelling uncertainties and from the fact that the experimental curve is included under the prediction without excessive overestimation it can be concluded as being a good estimate of the response output in the mid-frequency range.

\section{High-Frequency Predictions}

At higher frequencies, as already stated, the results obtained with or without the harness attached to the panel are very similar (i.e., the cable does not change significantly the response) and therefore no further analyses need to be implemented; in particular, predictions obtained through Finite Element Analysis are not reliable anymore and a statistical approach should be preferred.

Now, this methodology will be extended to the case of more complex structures with the introduction of a different approach to deal with the mid-frequency range aimed at replacing the use of the FMCS.

\section{Computational Approach for Structural Assemblies}

The issue of developing a method for modelling harnesses acquires importance in the context of complex structural assemblies. Figure 18, as an example, shows the considerable mass of cables that connect the various subsystems of the structure: they play a very important role in the vibration transmission path between the input and the output locations (i.e., the impedance of the cables will impact the loads and its attachment points on the cable).

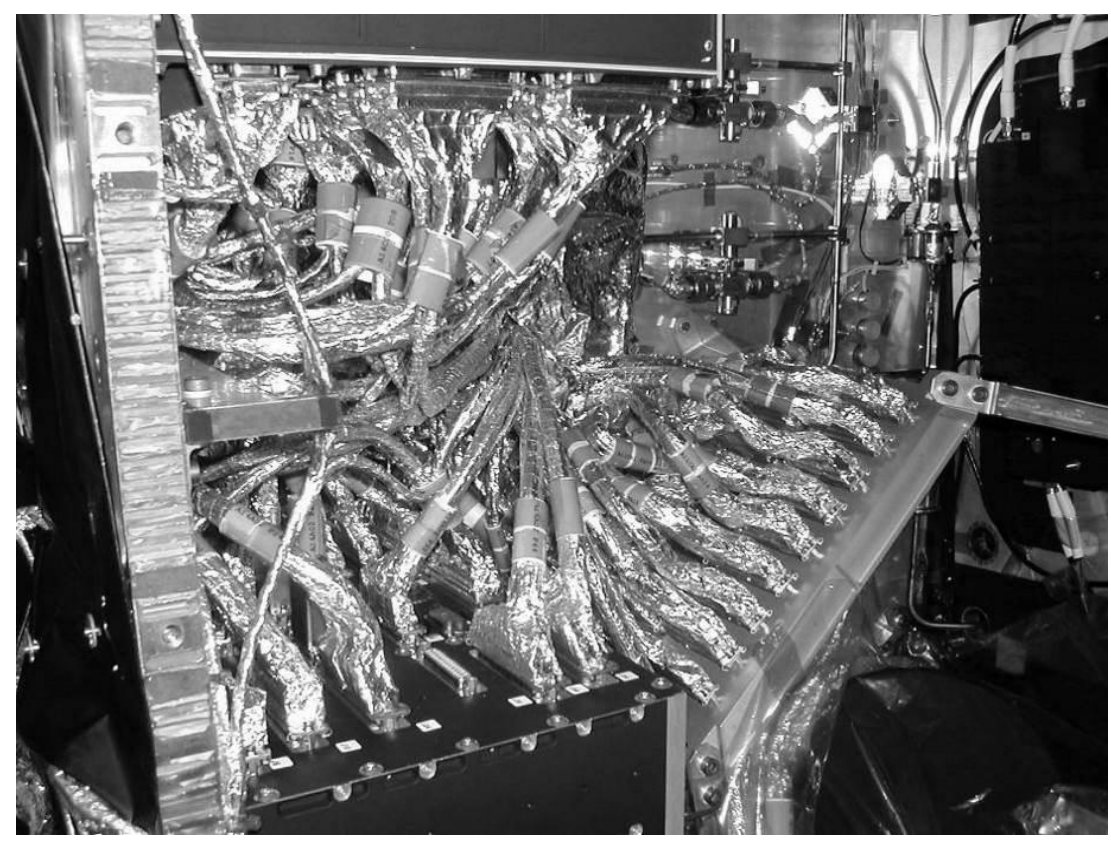

Figure 18: Harness inside the right wall of Mars Express ${ }^{1}$

\footnotetext{
${ }^{1}$ http://blogs.esa.int/mex/2014/02/21/space-is-really-really-big-except-sometimes-it-isnt/
} 
It was proven in the previous sections that the addition of a single cable can create significant differences in the final response. Figure 18 represents an extreme example, but it gives the idea of the potential magnitude of the problem. The effect of the cables cannot be neglected or significantly captured as non-structural mass.

For this kind of structure, the general methodology can still be applied: keeping the model unchanged for the high-frequency range and adding Non-Structural Mass and damping with addition of stiffness in the low-frequency range. For the mid-frequency range, a FMCS for a whole satellite to describe the effect of the harness would be computationally too expensive. There is therefore the need to find a method which enables the perturbation of the mass and stiffness properties without having to rerun the whole FEM several times, but which ultimately gives results as satisfying as the FMCS. What is proposed here is to apply a Monte Carlo Simulation on several reduced models, which were obtained after perturbing reduced subsystems through a Component Mode Synthesis approach. The advantages of doing this would be:

- Dealing with subsystems with just a few hundred modal coordinates instead of hundreds of thousands of physical coordinates, thus saving a considerable amount of computation time;

- Having the subsystems described in terms of natural frequencies and modal participation factors, and therefore being able to manipulate the stiffness and mass properties without having the physical model at disposal.

In the following section (Section V.A) the theory behind this method (named Craig-Bampton Stochastic Method, CBSM) will be described and in Section V.B a real industrial application will show how similar the CBSM predictions are to the FMCS predictions.

\section{A. Craig-Bampton Stochastic Method}

The Craig-Bampton Stochastic Method [23] basically consists of a stochastic approach of the Component Mode Synthesis [24]. The method is particularly suitable for the space industry as spacecraft structures' components are often delivered by subcontractors in the form of Craig-Bampton reduced models. For the convenience of the reader, the main steps of the Component Mode Synthesis are summarised below. The equation governing the dynamics of the whole systems can be written as:

$$
\mathbf{M x}+\mathbf{C} \dot{\mathbf{x}}+\mathbf{K x}=\mathbf{f}
$$

Where $\mathbf{M}, \mathbf{C}$ and $\mathbf{K}$ are respectively the mass, damping and stiffness matrices of the structure, $\mathbf{x}$ is the vector of the physical degrees of freedom and $\mathbf{f}$ is the vector of the forces. The whole model is now divided into subsystems with the corresponding degrees of freedom categorised into two groups: R (boundary, input and output degrees of freedom) and L (all the other degrees of freedom):

$$
\mathbf{x}_{\mathbf{i}}=\left(\begin{array}{c}
\mathbf{x}_{\mathbf{i R}} \\
\mathbf{x}_{\mathrm{iL}}
\end{array}\right)
$$


The $\mathrm{L}$ degrees of freedom can then be converted into a specific number of modal coordinates; the final response will then be reconstructed using only the modes which give significant contributions and therefore the number of modal coordinates will be significantly smaller than the number of physical degrees of freedom of the subsystem. The transformation matrix can therefore be set as:

$$
\mathbf{x}_{\mathrm{i}}=\left(\begin{array}{c}
\mathbf{x}_{\mathrm{iR}} \\
\mathbf{x}_{\mathrm{iL}}
\end{array}\right)=\left(\begin{array}{cc}
I & 0 \\
\phi_{\mathrm{iR}} & \phi_{\mathrm{iL}}
\end{array}\right)=\mathrm{CB}\left(\begin{array}{c}
\mathbf{x}_{\mathrm{iR}} \\
\mathbf{q}_{\mathrm{im}}
\end{array}\right)
$$

Where $\boldsymbol{\phi}_{\mathbf{i} \mathbf{R}}$ is a transformation matrix that relates rigid body physical displacements at the interface to physical displacements of the elastic degrees of freedom, $\mathbf{q}_{\mathbf{i m}}$ are the modal responses, $\boldsymbol{\phi}_{\mathbf{i L}}$ is a transformation matrix that relates the modal responses $\mathbf{q}_{\mathbf{i m}}$ to physical displacements of the elastic degrees of freedom, $\mathbf{C B}$ is the CraigBampton transformation matrix, $\boldsymbol{\phi}_{\mathbf{i R}} \mathbf{x}_{\mathbf{i R}}$ are the constrained node displacements and $\boldsymbol{\phi}_{\mathbf{i L}} \mathbf{q}_{\mathbf{i m}}$ are the normal mode displacements. Substituting these new coordinates into the equation of motion and pre-multiplying by the transpose of $\mathbf{C B}$, it yields:

$$
\begin{aligned}
& \left(\begin{array}{ll}
M_{i B B} & M_{i B m} \\
M_{i m B} & M_{i m m}
\end{array}\right)\left(\begin{array}{c}
\ddot{x_{i R}} \\
q_{i m}^{\ddot{i m}}
\end{array}\right)+\left(\begin{array}{ll}
C_{i B B} & C_{i B m} \\
C_{i m B} & C_{i m m}
\end{array}\right)\left(\begin{array}{c}
x_{i R}^{*} \\
q_{i m}^{*}
\end{array}\right)+ \\
& +\left(\begin{array}{ll}
\mathbf{K}_{\mathrm{iBB}} & \mathbf{K}_{\mathrm{iBm}} \\
\mathbf{K}_{\mathrm{imB}} & \mathbf{K}_{\mathrm{imm}}
\end{array}\right)\left(\begin{array}{c}
\mathrm{x}_{\mathrm{iR}} \\
\mathbf{q}_{\mathrm{im}}
\end{array}\right)=\left(\begin{array}{c}
\mathrm{f}_{\mathrm{iR}}+\phi_{\mathbf{R}}{ }^{\mathrm{T}} \mathrm{f}_{\mathrm{iL}} \\
\phi_{\mathbf{R}}{ }^{\mathrm{T}} \mathrm{f}_{\mathrm{iL}}
\end{array}\right)
\end{aligned}
$$

Where, for mass normalised matrices, $\mathbf{M}_{\mathbf{i B B}}$ is the structural mass matrix reduced to the boundary nodes, $\mathbf{M}_{\mathbf{i B m}}$ is the matrix of the modal participation factors and $\mathbf{M}_{\mathbf{i m B}}$ is the transpose of $\mathbf{M}_{\mathbf{i B m}} \cdot \mathbf{C}_{\mathbf{i B B}}, \mathbf{C}_{\mathbf{i B m}}$ and $\mathbf{C}_{\mathbf{i m B}}$ are all equal to $0 ; \mathbf{C}_{\mathbf{i m m}}$ contains the modal damping values for each of the modal coordinates considered; $\mathbf{K}_{\mathbf{i B m}}$ and $\mathbf{K}_{\mathbf{i m B}}$ are also equal to 0 and $\mathbf{K}_{\mathbf{i m m}}$ contains the natural frequencies of the subsystems with the boundary degrees of freedom constrained. Substituting the terms described above in equation 4, the mass, damping and stiffness matrices become:

$$
\mathbf{M}_{\mathrm{i}}=\left[\begin{array}{cc}
\mathbf{M}_{\mathrm{iBB}} & \widetilde{\mathbf{M}_{1 B m}} \\
\widetilde{\mathbf{M}_{1 \mathrm{BB}}} & \mathbf{I}
\end{array}\right] \quad \mathbf{C}_{\mathrm{i}}=\left[\begin{array}{cc}
0 & 0 \\
0 & 2 \xi\left(\widetilde{\omega_{10}}\right)
\end{array}\right] \quad \mathbf{K}_{\mathrm{i}}=\left[\begin{array}{cc}
\mathbf{K}_{\mathrm{iBB}} & 0 \\
0 & \left(\widetilde{\omega_{10}}\right)^{2}
\end{array}\right]
$$

Where the accent identifies the terms which will be perturbed.

The process of reducing with Craig-Bampton is repeated for all the subsystems; the corresponding mass, damping and stiffness matrices are then merged together to rebuild the overall model of the structure. Depending on how many perturbations need to be applied, a certain number of models is obtained, which are then run to obtain the responses and a statistical manipulation is finally performed to obtain a final response. This method represents an 
extension of the work done by Mace and Shorter [25]. Applying the aforementioned method it was found that perturbations of only resonance frequencies of the subsystems were not sufficient to capture all the changes produced by the FMCS.

In order to prove the validity of the CBSM, the method was applied to predict the response for the TK_loc1 configuration. The panel was divided into 4 subsystems (a quarter of the panel each) and the natural frequencies and the modal participation factors of each subsystem were randomised. The result is shown in Figure 19. For both predictions, the average summed to the standard deviation twice was chosen to obtain a $95 \%$ confidence final upper envelope.

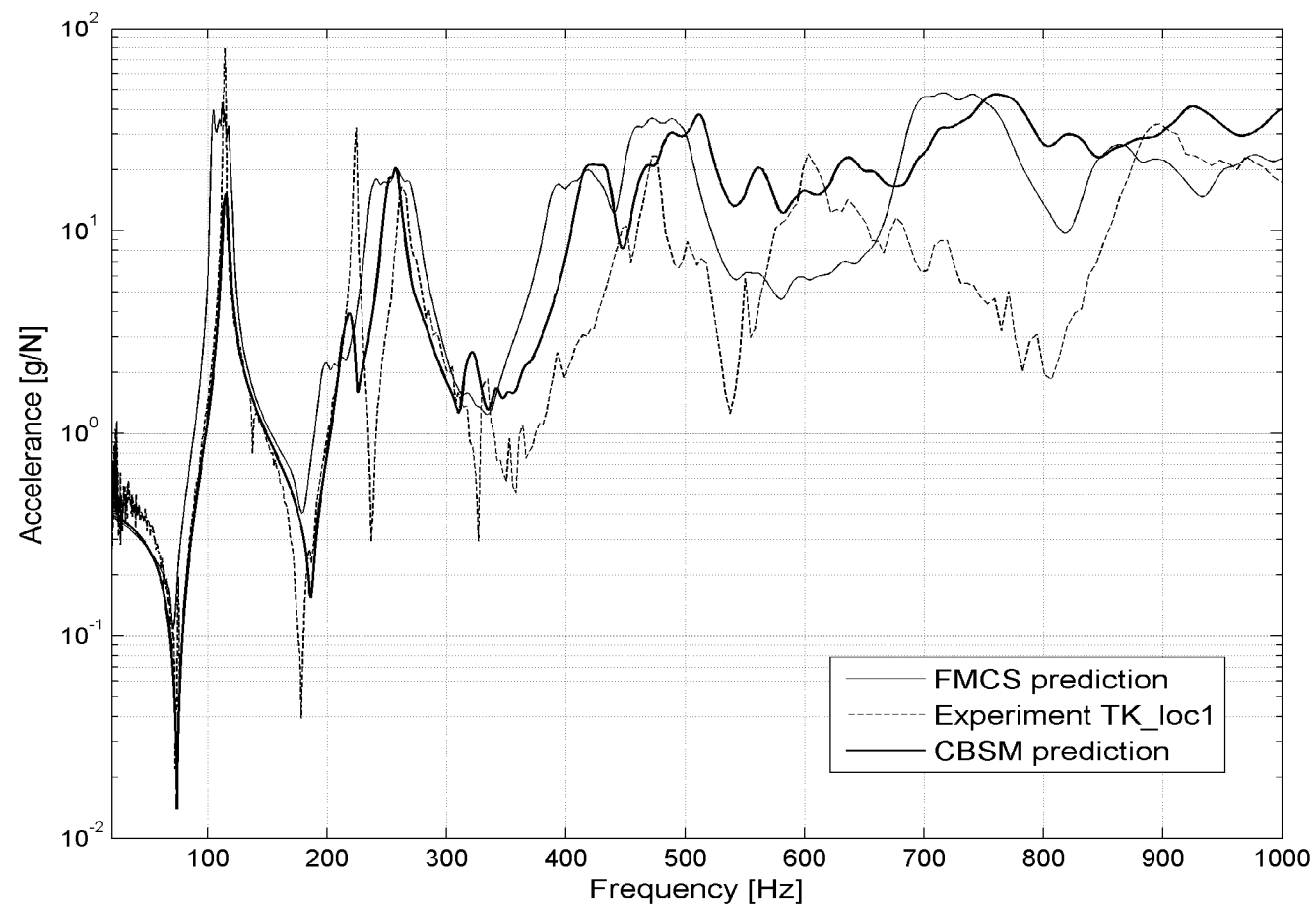

Figure 19: FMCS and CBSM applied for the configuration TK_loc1

As Figure 19 shows, the CBSM actually improves the prediction (comparing it to the experimental results) in a few segments of the considered frequency range.

\section{B. Industrial application}

The advantages of using the CBSM apply especially to complex industrial applications. Here, the Structural Qualification Model (SQM) of the satellite SSTL 300-S1 is considered (Figure 20). The structure is essentially an assembly of aluminium honeycomb panels connected by brackets to generate a box like structure which supports a CFRP telescope (the cylindrical structure visible in Figure 20, protruding from the top of the assembly). The model, which was built using Patran and solved using Nastran, has a mass of $410 \mathrm{Kg}$ and includes 621795 nodes and 568177 elements. More details on the spacecraft can be found in literature [26, 27]. The FEM of the satellite was analysed both with a FMCS and a CBSM. 

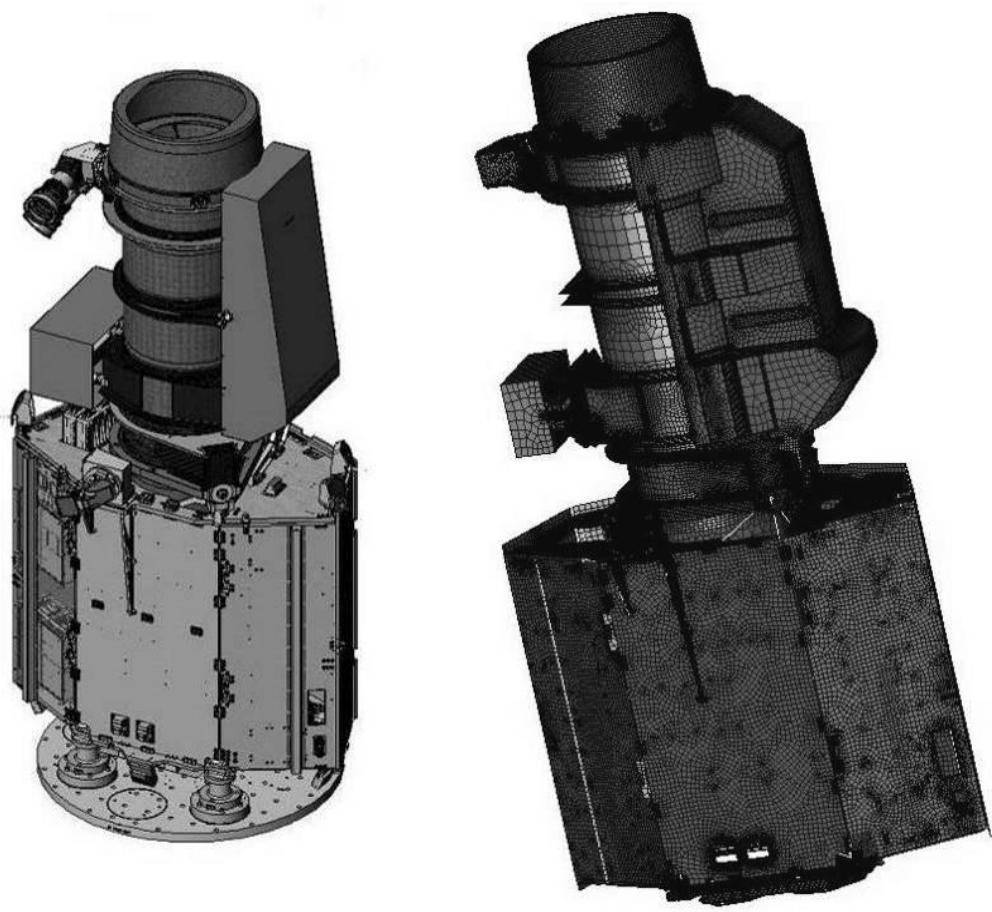

Figure 20: Satellite SSTL 300-S1 with FE Model

As for the CBSM, the structure was divided into 5 different subsystems and each one of them was reduced with the Craig-Bampton reduction. Mass, damping and stiffness matrices were obtained for each subsystem. The natural frequencies of the subsystems constrained in the chosen boundary degrees of freedom and the correspondent modal participation factors were perturbed.

The process of perturbing the subsystems and building the final matrices for the whole structure was repeated 100 times. The final stochastic model was computed, using the 100 different models that were built with the CBSM. Input and output locations were kept as physical degrees of freedom throughout the process. Working with this stochastic model a significant reduction can be observed in the computational effort involved, as now the runs are performed using a model which has just a few hundred modal coordinates, compared to the initial FE model, which had hundreds of thousands physical coordinates. The CBSM is now compared to the prediction given by a FMCS. Gaussian perturbations (with $\sigma$ value of 5\%) of the main materials of the structure, of the stiffness of the joints and of the materials were applied; 100 different models were run and a final stochastic model was built.

Figure 21 shows the comparison between the transfer functions computed with the use of the stochastic models built both with the CBSM and with the FMCS. Again, for both predictions, the average summed to the standard deviation twice was chosen to obtain a 95\% confidence final upper envelope. Looking at the mid-frequency range, which is in particular the range of interest (given that the low-frequency range is already well predicted by the nominal FE analysis), it can be seen that the two predictions are close to each other. Since FMCS is considered the benchmark method for analysis including uncertainties, the fact that CBSM gives predictions very similar to FMCS confirms the quality of the CBSM, which works with much less computational effort. In particular, Table 3 lists the computational times involved using an Intel Core i7-2600, CPU @3.40 GHz and RAM 16 Gb. 


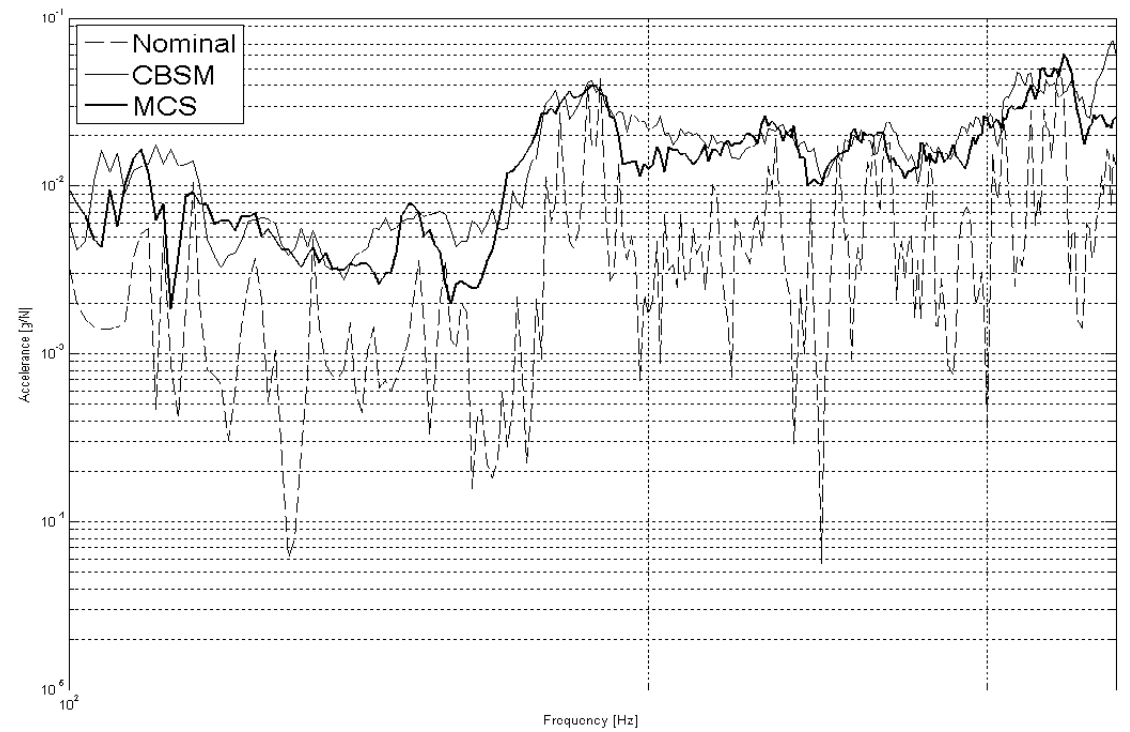

Figure 21: CBSM and FMCS predictions compared to the nominal solution

Table 3: Time computations of CBSM and FMCS.

CBSM

Run of 1 model
Run of 50 models
Implementation of the method*

$0 \min 16 \mathrm{sec}$

$13 \min 33 \mathrm{sec}$

$\sim 19 \mathrm{~min}$
FMCS

\author{
$21 \mathrm{~min} 13 \mathrm{sec}$ \\ 35 h $21 \min 40 \mathrm{sec}$ \\ $\sim 35$ h 30 min
}

*It includes the time used to run the Matlab codes used for reading the results' files and the statistical manipulation of the data.

\section{Conclusions}

In this article, the influence of harnesses on spacecraft structures was investigated through an experimental campaign involving a honeycomb panel, which was tested in various configurations (bare and with two different cables in two different locations). The results showed that the effect of the harness depended on the frequency range and the amplitude of the input, proving the limitation of using non-structural mass to represent harness.

The analysis was tackled from three different viewpoints. In the low-frequency range non-structural mass was added with an addition of stiffness and damping. In the mid-frequency range securing harness on the cable caused several changes and a Full Monte Carlo Simulation has been chosen to produce a response prediction, including an indication of the scatter of results that should be expected. In the high-frequency range the responses obtained with and without the harness were similar and this suggested the use of the same technique to be used in both cases.

The aforementioned general methodology was successfully applied to the case of the honeycomb panel tested and finally a less computationally expensive method (Craig-Bampton Stochastic Method) was described, developed and applied to an industrial application; this method is aimed at replacing the Full Monte Carlo Simulation for complex structures. 


\section{References}

[1] S. Rybak, R. Mayo, S. Lieberman, and L. Hartter, "Ultrahigh-accuracy body-pointing system for the Large Space Telescope," Journal of Spacecraft and Rockets, vol. 13, pp. 220-228, 1976.

[2] C. L. Foster, M. L. Tinker, G. S. Nurre, and W. A. Till, "Solar-Array Induced Disturbance of the Hubble Space Telescope Pointing System," Journal of Spacecraft and Rockets, vol. 32, pp. 634-644, 1995.

[3] J. M. Le Duigou, "Microvibration Measurements on Spot 4, Results of the Micromedy Experiment," in Proceedings of a European Conference on Spacecraft Structures, Material and Mechanical Testing, Braunschweig, Germany, ESA, 1998.

[4] M. Privat, "On Ground and in Orbit Microvibration Measurement Comparison," in Space Mechanisms and Tribology, Proceedings of the 8th European Symposium, Toulouse, France, European Space Agency, 1999.

[5] J. Choi and D. J. Inman, "Spectrally Formulated Modeling of a Cable-Harnessed Structure," Journal of Sound and Vibration, vol. 333, pp. 3286-3304, 2014.

[6] W. Desmet, B. Pluymers, and O. Atak, "Mid-Frequency" - CAE Methodologies for Mid-Frequency Analysis in Vibrations and Acoustics, 2012.

[7] Z. Zhang, G. S. Aglietti, and W. Zhou, "Microvibrations induced by a cantilevered wheel assembly with a softsuspension system," AIAA Journal, vol. 49, pp. 1067-1079, 2011.

[8] Z. Zhang, G. S. Aglietti, and W. J. Ren, "Microvibration Model Development and Validation of a Cantilevered Reaction Wheel Assembly," Applied Mechanics and Materials, vol. 226, pp. 133-137, 2012.

[9] G. Aglietti, S. Gabriel, R. Langley, and E. Rogers, "A modeling technique for active control design studies with application to spacecraft microvibrations," The Journal of the Acoustical Society of America, vol. 102, p. $2158,1997$.

[10] G. Aglietti, R. Langley, E. Rogers, and S. Gabriel, "An efficient model of an equipment loaded panel for active control design studies," The Journal of the Acoustical Society of America, vol. 108, p. 1663, 2000.

[11] O. Takahara, N. Yoshida, H. Uchida, and K. Komatsu, "Study of estimation method of microvibration with simulated satellite structure " presented at the IAC, Glasgow, UK, 2008.

[12] N. Yoshida, "Issues on dynamic interface between Optical mission payload and spacecraft Bus," presented at the 28th international symposium on space technology and science, Okinawa, Japan, 2011.

[13] N. Yoshida, "Comparison and implication of statistical combination methods in pointing error analysis," presented at the 24th International symposium on space technology and science, Miyazaki, Japan, 2004.

[14] T. Shimizu, S. Nagata, S. Tsuneta, T. Tarbell, C. Edwards, R. Shine, et al., "Image stabilization system for Hinode (Solar-B) solar optical telescope," Solar Physics, vol. 249, pp. 221-232, 2008.

[15] A. H. Pevrot and A. M. Goulois, "Analysis of Cable Structures," Computers \& Structures, vol. 10, pp. 805-813, 1979.

[16] C.-S. Kao and C.-H. Kou, "The Influence of Broken Cables on the Structural Behavior of Long-Span Cable-Stayed Bridges," Journal of Marine Science and Technology, vol. 18, pp. 395-404, 2010.

[17] A. M. Abdel-Ghaffar and M. A. Khalifa, "Importance of Cable Vibration in Dynamics of Cable-Stayed Bridges," Journal of Engineering Mechanics, vol. 117, pp. 2571-2589, 1991.

[18] K. S. Spak, G. S. Agnes, and D. J. Inman, "Bakeout Effects on Dynamic Response of Spaceflight Cables," Journal of Spacecraft and Rockets, 2014.

[19] J. Choi and D. J. Inman, "Development of Predictive Modeling for Cable Harnessed Structure," presented at the 54th AIAA/ASME/ASCE/AHS/ASC Structures, Structural Dynamics, and Materials Conference, Boston, 2013.

[20] V. Babuska, D. M. Coombs, J. C. Goodding, E. V. Ardelean, L. M. Robertson, and S. A. Lane, "Modeling and Experimental Validation of Space Structures with Wiring Harnesses," Journal of Spacecraft and Rockets, vol. 47, pp. 1038-1052, 2010.

[21] J. C. Goodding, E. V. Ardelean, V. Babuska, L. M. Robertson, and S. A. Lane, "Experimental Techniques and Structural Parameter Estimation Studies of Spacecraft Cables," Journal of Spacecraft and Rockets, vol. 48, pp. 942957, 2011.

[22] D. M. Coombs, J. C. Goodding, V. Babuska, E. V. Ardelean, L. M. Robertson, and S. A. Lane, "Dynamic Modeling and Experimental Validation of a Cable-Loaded Panel," Journal of Spacecraft and Rockets, vol. 48, pp. 958-973, 2011.

[23] M. Remedia, G. S. Aglietti, G. Richardson, B. LePage, and T. Seabrook, "Application of a Substructural Method for Modelling Satellite Microvibrations," presented at the European Conference on Spacecraft Structures, Materials \& Environmental Testing, Braunschweig, Germany, 2014.

[24] J. Wijker, "Component Mode Synthesis," in Mechanical Vibrations in Spacecraft Design, ed: Springer Berlin Heidelberg, 2004, pp. 369-398.

[25] B. Mace and P. Shorter, "A local modal/perturbational method for estimating frequency response statistics of built-up structures with uncertain properties," Journal of Sound and Vibration, vol. 242, pp. 793-811, 2001.

[26] C. Hamar, T. Wood, S. Alsami, and B. Hallett, "Development of the SSTL-300-S1 composite imager barrel structure," in 13th European Conference on Spacecraft Structures, Materials \& Environmental Testing (SSMET), Braunschweig, Germany, 2014.

[27] G. Richardson, G. Smet, G. S. Aglietti, T. Seabrook, and S. Alsami, "Managing micro-vibration on the SSTL-300 S1 a $400 \mathrm{~kg} 1 \mathrm{~m}$ resolution Earth imaging satellite," in 13th European Conference on Spacecraft Structures, Materials \& Environmental Testing (SSMET), Braunschweig, Germany, 2014. 
